\title{
Palladium-Catalyzed Oxidative Cyclizations: Synthesis of Dihydropyranones and Furanones
}

Maud Reiter, Hazel Turner, Rebecca Mills-Webb and Véronique Gouverneur*

\section{Contents}

Experimental

page $\mathrm{S} 2$

Characterization of compounds 1b-d, 1f-g, 2c-g, 3b, 3d-f, 4b-f, 6, 7, 10, 12-14.

X-ray Crystallography data for 11

page $\mathrm{S} 10$

${ }^{1} \mathrm{H}$ NMR spectra

page S16

${ }^{13} \mathrm{C}$ NMR spectra

page $\mathrm{S} 40$

References

page S54 


\section{Experimental}

syn-1-Hydroxy-1-(4-methoxy-phenyl)-2-methyl-hex-4-en-3-one (1b). $72 \%$ yield, with $d e>99 \%$, as a colourless oil; $v_{\max }\left(\mathrm{film} / \mathrm{cm}^{-1}\right): 3418$ and $1651 ; \delta_{H}(400 \mathrm{MHz}$, $\left.\mathrm{CDCl}_{3}\right) 1.08(3 \mathrm{H}, \mathrm{d}, J 7.5 \mathrm{~Hz}), 1.91(3 \mathrm{H}, \mathrm{dd}, J 6.8$ and $1.7 \mathrm{~Hz}), 3.01(1 \mathrm{H}, \mathrm{qd}, J 7.5$ and $3.4 \mathrm{~Hz}), 3.39(1 \mathrm{H}, \mathrm{d}, J$ 2.1 Hz), $3.81(3 \mathrm{H}, \mathrm{s}), 5.06(1 \mathrm{H}, \mathrm{dd}, J 3.4$ and $2.1 \mathrm{~Hz}), 6.12(1 \mathrm{H}$, d, $J 15.7 \mathrm{~Hz}), 6.89(2 \mathrm{H}, \mathrm{d}, J \quad 8.8 \mathrm{~Hz}), 6.93(1 \mathrm{H}, \mathrm{dq}, J 15.7$ and $6.8 \mathrm{~Hz}), 7.27(2 \mathrm{H}, \mathrm{d}, J$ $8.8 \mathrm{~Hz}) ; \delta_{C}\left(100 \mathrm{MHz}, \mathrm{CDCl}_{3}\right)$ 10.6, 18.4, 49.6, 55.3, 72.6, 113.6, 127.1, 128.8, 130.6, 144.2, 173.7, 205.1; $m / z 234.1260\left(\mathrm{C}_{14} \mathrm{H}_{18} \mathrm{O}_{3}\right.$ requires 234.1256).

syn-6-Hydroxy-5-methyl-8-phenyl-oct-2en-4-one (1c). $71 \%$ yield, de > 99\%, as a colouless oil; $v_{\max }\left(\right.$ film $\left./ \mathrm{cm}^{-1}\right) 3443,2937,1682,1651,1625 ; \delta_{H}\left(400 \mathrm{MHz}, \mathrm{CDCl}_{3}\right) 1.17$ $(3 \mathrm{H}, \mathrm{d}, J$ 7.2 Hz), $1.63(2 \mathrm{H}, \mathrm{ddddd}, J 13.6,9.9,6.5,3.8$, and $1.0 \mathrm{~Hz}), 1.87(1 \mathrm{H}, \mathrm{dd}, J 9.2$ and $5.5 \mathrm{~Hz}) 1.91(3 \mathrm{H}, \mathrm{dd}, J 6.8$ and $1.5 \mathrm{~Hz}), 2.67(1 \mathrm{H}$, ddd, $J$ 14.0, 9.6 and $6.8 \mathrm{~Hz}), 2.77$ $(1 \mathrm{H}, \mathrm{qd}, J$ 7.2 and $2.7 \mathrm{~Hz}), 2.87(1 \mathrm{H}, \mathrm{ddd}, J$ 14.0, 9.6 and 5.1 Hz), 3.07-3.09 (1H, m), $6.15(1 \mathrm{H}, \mathrm{dq}, J 15.7$ and $1.7 \mathrm{~Hz}), 6.91(1 \mathrm{H}, \mathrm{dq}, J 15.4$ and $6.8 \mathrm{~Hz}), 7.17-7.32(5 \mathrm{H}, \mathrm{m}) ; \delta_{C}$ $\left(100 \mathrm{MHz}, \mathrm{CDCl}_{3}\right)$ 10.4, 14.4, 18.4, 32.1, 32.3, 35.9, 36.8, 47.3, 48.2, 70.3, 73.1, 125.8, 128.4, 128.5, 131.3, 142.0, 144.2, 205.1; $\mathrm{m} / \mathrm{z} 233.1546\left(\mathrm{C}_{15} \mathrm{H}_{20} \mathrm{O}_{2}\right.$ requires 233.1542).

syn-6-Hydroxy-5,7,7-trimethyl-oct-2-en-4-one (1d). 64\% yield, de > 99\%, as a colouless oil; $v_{\max }\left(\mathrm{film} / \mathrm{cm}^{-1}\right) 3484,2957,1691,1627 ; \delta_{H}\left(400 \mathrm{MHz}, \mathrm{CDCl}_{3}\right) 0.95(9 \mathrm{H}$, s), $1.17(3 \mathrm{H}, \mathrm{d}, J 6.8 \mathrm{~Hz}), 1.93(3 \mathrm{H}, \mathrm{dd}, J 6.8$ and $1.7 \mathrm{~Hz}), 2.56(1 \mathrm{H}, \mathrm{d}, J 3.7 \mathrm{~Hz}), 3.04$ $(1 \mathrm{H}, \mathrm{qd}, J 7.1$ and $3.4 \mathrm{~Hz}), 3.61(1 \mathrm{H}, \mathrm{dd}, J 3.7$ and $3.4 \mathrm{~Hz}), 6.16(1 \mathrm{H}, \mathrm{dq}, J 15.7$ and 1.5 $\mathrm{Hz}), 6.94(1 \mathrm{H}, \mathrm{dq}, J 15.7$ and $7.1 \mathrm{~Hz}) ; \delta_{C}\left(100 \mathrm{MHz}, \mathrm{CDCl}_{3}\right)$ 9.9, 18.3, 19.0, 30.5, 44.7, $76.3,130.4,143.9,205.1$. 
$61 \%$ yield, as a colourless oil $(0.6 \mathrm{~g}, 3 \mathrm{mmol})$, with $d e=50 \% ; v_{\max }\left(\mathrm{film} / \mathrm{cm}^{-1}\right): 3419$ and $1650 ; \delta_{H}\left(400 \mathrm{MHz}, \mathrm{CDCl}_{3}\right) 0.84(3 \mathrm{H}, \mathrm{d}, J 7.5 \mathrm{~Hz}), 1.81(3 \mathrm{H}, \mathrm{dd}, J 7.1$ and $1.6 \mathrm{~Hz})$, $3.08(1 \mathrm{H}, \mathrm{dq}, J 8.1$ and $7.3 \mathrm{~Hz}), 3.49(1 \mathrm{H}, \mathrm{m}), 3.81(3 \mathrm{H}, \mathrm{s}), 4.69(1 \mathrm{H}, \mathrm{d}, J 8.3 \mathrm{~Hz}), 6.12$ $(1 \mathrm{H}, \mathrm{dq}, J 15.7$ and $1.3 \mathrm{~Hz}), 6.89(2 \mathrm{H}, \mathrm{d}, J 8.8 \mathrm{~Hz}), 6.88(1 \mathrm{H}, \mathrm{dq}, J 15.7$ and $6.8 \mathrm{~Hz})$, $7.27(2 \mathrm{H}, \mathrm{d}, J 8.8 \mathrm{~Hz}) ; \delta_{C}\left(100 \mathrm{MHz}, \mathrm{CDCl}_{3}\right)$ 14.7, 18.3, 49.6, 50.4, 72.6, 113.6, 127.8, 131.9, 134.5, 144.0, 159.0, 204.2; $\mathrm{m} / \mathrm{z} 234.1260\left(\mathrm{C}_{14} \mathrm{H}_{18} \mathrm{O}_{3}\right.$ requires 234.1256).

(E)-anti-6-Hydroxy-5,7-dimethyloct-2-en-4-one (1g). de $=78 \% ; \quad v_{\max }\left(\mathrm{film} / \mathrm{cm}^{-1}\right)$ : 3443, 1644; $\delta_{H}\left(400 \mathrm{MHz}, \mathrm{CDCl}_{3}\right) 0.93(3 \mathrm{H}, \mathrm{d}, J 1.5 \mathrm{~Hz}), 0.95(3 \mathrm{H}, \mathrm{d}, J 1.5 \mathrm{~Hz}), 1.17$ (3H, dd, $J 7.3 \mathrm{~Hz}), 1.75(1 \mathrm{H}, \mathrm{m}), 1.93(3 \mathrm{H}, \mathrm{dd}, J 6.8$ and $1.5 \mathrm{~Hz}), 2.85(1 \mathrm{H}, \mathrm{d}, J 7.3 \mathrm{~Hz})$, $3.0(1 \mathrm{H}, \mathrm{qd}, J 7.3$ and $6.0 \mathrm{~Hz}), 3.44(1 \mathrm{H}, \mathrm{q}, J 5.8 \mathrm{~Hz}), 6.19(1 \mathrm{H}, \mathrm{dq}, J 15.7$ and $1.5 \mathrm{~Hz})$, $6.94(1 \mathrm{H}, \mathrm{dq}, J 15.7$ and $6.8 \mathrm{~Hz}) ; \delta_{C}\left(100 \mathrm{MHz}, \mathrm{CDCl}_{3}\right)$ 14.1, 18.3, 19.0, 19.9, 31.1, 45.5, 78.8, 131.9, 143.9, 205.5; $\mathrm{m} / z$ 171.1377 $\left(\mathrm{C}_{10} \mathrm{H}_{19} \mathrm{O}_{2}\right.$ requires 171.1385).

(syn)-3,6-Dimethyl-2-phenethyl-2,3-dihydro-pyran-4-one (2c). 68\% yield, de > 99\%; $v_{\max }\left(\mathrm{film} / \mathrm{cm}^{-1}\right) 1669,1614 ; \delta_{H}\left(400 \mathrm{MHz}, \mathrm{CDCl}_{3}\right) 1.07(3 \mathrm{H}, \mathrm{d}, J 7.3 \mathrm{~Hz}), 1.83(1 \mathrm{H}$, dddd, $J$ 14.1, 9.4, 7.0 and $4.7 \mathrm{~Hz}), 2.0(3 \mathrm{H}, \mathrm{s}), 2.17(1 \mathrm{H}$, dddd, $J$ 12.6, 8.1, 9.3 and 5.8 $\mathrm{Hz}), 2.29(1 \mathrm{H}, \mathrm{dq}, J 7.6$ and $3.3 \mathrm{~Hz}), 2.73(1 \mathrm{H}, \mathrm{ddd}, J 13.9,8.8$ and $7.1 \mathrm{~Hz}), 2.84(1 \mathrm{H}$, ddd, $J$ 14.7, 9.6 and $5.8 \mathrm{~Hz}), 4.31(1 \mathrm{H}$, ddd, $J$ 7.8, 4.3 and $3.3 \mathrm{~Hz}), 5.25(1 \mathrm{H}, \mathrm{s}), 7.19-$ $7.34(5 \mathrm{H}, \mathrm{m}) ; \delta_{C}\left(100 \mathrm{MHz}, \mathrm{CDCl}_{3}\right)$ 9.7, 20.8, 31.5, 31.9, 42.7, 80.8, 103.4, 126.2, 128.4, 128.5, 140.8, 173.5, 197.4; $\mathrm{m} / \mathrm{z} 231.1385\left(\mathrm{C}_{15} \mathrm{H}_{18} \mathrm{O}_{2}\right.$ requires 231.1385).

syn-2-tert-Butyl-3,6-dimethyl-2,3-dihydro-pyran-4-one (2d). Purification by silica gel chromatography (1:4; EtOAc: hexane) gave $\mathbf{2 d}$ as a colourless oil in 55\% yield, de > $99 \% ; v_{\max }\left(\mathrm{film} / \mathrm{cm}^{-1}\right) 1661 ; \delta_{H}\left(400 \mathrm{MHz}, \mathrm{CDCl}_{3}\right) 1.07$ (9H, s), $1.12(3 \mathrm{H}, \mathrm{d}, J 7.3 \mathrm{~Hz})$, 
$2.03(3 \mathrm{H}, \mathrm{s}), 2.44(1 \mathrm{H}$, qdd, $J$ 7.3, 2.8 and $1.2 \mathrm{~Hz}), 3.80(1 \mathrm{H}, \mathrm{dd}, J 10.1$ and $2.7 \mathrm{~Hz})$, $5.23(1 \mathrm{H}, \mathrm{s}) ; \delta_{C}\left(100 \mathrm{MHz}, \mathrm{CDCl}_{3}\right)$ 11.7, 20.6, 27.0, 34.3, 41.7, 88.5, 103.0, 174.6, 198.6; $m / z 183.1393\left(\mathrm{C}_{11} \mathrm{H}_{19} \mathrm{O}_{2}\right.$ requires 183.1385).

anti-3,6-Dimethyl-2-benzyl-2,3-dihydro-pyran-4-one (2e). Purification by silica gel chromatography (1:3, EtOAc: hexane) gave $2 \mathbf{e}$ as a colourless oil in $50 \%$ yield, de > $99 \% ; v_{\max }\left(\mathrm{film} / \mathrm{cm}^{-1}\right) 1668,1615 ; \delta_{H}\left(400 \mathrm{MHz}, \mathrm{CDCl}_{3}\right) 0.92(3 \mathrm{H}, \mathrm{d}, J 6.8 \mathrm{~Hz}), 2.04(3 \mathrm{H}$, s), $2.52(1 \mathrm{H}, \mathrm{q}, J 6.8 \mathrm{~Hz}), 4.94(1 \mathrm{H}, \mathrm{d}, J 13.1 \mathrm{~Hz}), 5.43(1 \mathrm{H}, \mathrm{s}), 7.38-7.45(5 \mathrm{H}, \mathrm{m}), \delta_{C}$ (100 MHz, $\left.\mathrm{CDCl}_{3}\right)$ 10.3, 21.0, 43.8, 86.9, 104.4, 127.4, 128.7 ,129.0, 137.5, 173.4, 194.6; $m / z 202.1069\left(\mathrm{C}_{13} \mathrm{H}_{14} \mathrm{O}_{2}\right.$ requires 202.1072).

anti-3,6-Dimethyl-2-(4-methoxy-phenyl)-2,3-dihydro-pyran-4-one (2f). Purification by silica gel chromatography (1:4, EtOAc: hexane) gave $\mathbf{2 f}$ as a colourless oil in $82 \%$ yield, $d e=58 \% ; v_{\max }\left(\mathrm{film} / \mathrm{cm}^{-1}\right) 1659,1615 ; \delta_{H}\left(400 \mathrm{MHz}, \mathrm{CDCl}_{3}\right) 0.91(3 \mathrm{H}, \mathrm{d}, J$ $7.4 \mathrm{~Hz}), 2.02(3 \mathrm{H}, \mathrm{s}), 2.71(1 \mathrm{H}, \mathrm{dq}, J 13.8$ and $7.5 \mathrm{~Hz}), 3.82(3 \mathrm{H}, \mathrm{s}), 4.84(1 \mathrm{H}, \mathrm{d}, J 13.8$ Hz), $5.40(1 \mathrm{H}, \mathrm{s}) 6.97(2 \mathrm{H}, \mathrm{d}, J 8.8 \mathrm{~Hz}), 7.38(2 \mathrm{H}, \mathrm{d}, J 8.8 \mathrm{~Hz}) ; \delta_{C}\left(100 \mathrm{MHz}, \mathrm{CDCl}_{3}\right)$ $10.3,21.0,43.8,55.3,86.5,104.3,114.1,126.9,128.8,160.1,173.5,194.89 ; \mathrm{m} / \mathrm{z}$ $233.1180\left(\mathrm{C}_{14} \mathrm{H}_{17} \mathrm{O}_{3}\right.$ requires 233.1178).

anti-2-Isopropyl-3,6-dimethyl-2,3-dihydro-pyran-4-one (2g). Purification by silica gel chromatography (1:3, EtOAc: hexane) gave $\mathbf{2 g}$ as a colourless oil in $30 \%$ yield over 2 steps, $d e=70 \%$; $v_{\max }\left(\mathrm{film} / \mathrm{cm}^{-1}\right) 1671,1620 ; \delta_{H}\left(400 \mathrm{MHz}, \mathrm{CDCl}_{3}\right) 0.91(3 \mathrm{H}, \mathrm{d}, J 6.8$ Hz), 0.97 (3H, d, J 6.6 Hz), 1.09 (3H, d, J $6.8 \mathrm{~Hz}), 1.99$ (3H, s), 2.00-2.04 (1H, m), 2.40$2.55(1 \mathrm{H}, \mathrm{m}), 3.87(1 \mathrm{H}, \mathrm{dd}, J 11.8$ and $3.2 \mathrm{~Hz}), 5.29(1 \mathrm{H}, \mathrm{s}) ; \delta_{C}\left(100 \mathrm{MHz}, \mathrm{CDCl}_{3}\right) 10.3$, 15.0, 19.5, 20.8, 28.6, 40.6, 87.8, 103.6, 173.3, 195.7; m/z $169.1227\left(\mathrm{C}_{10} \mathrm{H}_{17} \mathrm{O}_{2}\right.$ requires 169.1229). 
(E)-4-Hydroxy-1-(4-methoxyphenyl)-4-methylpent-1-en-3-one $\quad(3 b) .^{1} \quad$ From $3-$ hydroxy-3-methyl-2-butanone (1 ml, $10 \mathrm{mmol})$, LiOH.H $\mathrm{H}_{2} \mathrm{O}(63 \mathrm{mg}, 1.9 \mathrm{mmol})$ and anisaldehyde $(2.38 \mathrm{ml}, 19.6 \mathrm{mmol})$ in $\mathrm{MeOH}(20 \mathrm{ml})$ and $\mathrm{H}_{2} \mathrm{O}(75 \mathrm{ml})$. Purification by silica gel chromatography (1:6, EtOAc: hexane) gave 3b in $46 \%$ yield as a pale yellow oil; $v_{\max }\left(\mathrm{film} / \mathrm{cm}^{-1}\right) 3419,1646 ; \delta_{H}\left(400 \mathrm{MHz}, \mathrm{CDCl}_{3}\right) 1.44(6 \mathrm{H}, \mathrm{s}), 3.87(3 \mathrm{H}, \mathrm{s}), 6.88$ $(1 \mathrm{H}, \mathrm{d}, J 15.4 \mathrm{~Hz}), 6.92(2 \mathrm{H}, \mathrm{m}), 7.56(2 \mathrm{H}, \mathrm{m}), 7.81(1 \mathrm{H}, \mathrm{d}, J 15.4 \mathrm{~Hz}) ; \delta_{C}(100 \mathrm{MHz}$, $\left.\mathrm{CDCl}_{3}\right)$ 26.6, 55.4, 75.4, 114.4, 115.8, 126.9, 130.5, 145.4, 162.0, 202.4; $\mathrm{C}_{15} \mathrm{H}_{15} \mathrm{O}_{3}$ found 219.04 .

(E)-2-Hydroxy-2-methyl-hept-4-en-3-one $\quad(3 d){ }^{2} \quad$ From 3-hydroxy-3-methyl-2butanone $(1 \mathrm{ml}, 10 \mathrm{mmol})$, propanal $(0.48 \mathrm{ml}, 6.6 \mathrm{mmol}),{ }^{\mathrm{n}} \mathrm{BuLi}(2.3 \mathrm{M}$ solution in hexanes, $8.5 \mathrm{ml}, 17 \mathrm{mmol})$ and diisopropylamine $(2.8 \mathrm{ml}, 17 \mathrm{mmol})$ in THF (40 ml) to give 2,5-dihydroxy-2-methylheptan-3-one $(0.72 \mathrm{~g}, 3 \mathrm{mmol})$ in $50 \%$ yield. The dehydration employed $\mathrm{CeCl}_{3} .7 \mathrm{H}_{2} \mathrm{O}(1.0 \mathrm{~g}, 2.8 \mathrm{mmol}), \mathrm{NaI}(0.42 \mathrm{~g}, 2.8 \mathrm{mmol})$ and 2,5dihydroxy-2-methylheptan-3-one $(0.3 \mathrm{~g}, 1.9 \mathrm{mmol})$ in anhydrous $\mathrm{MeCN}$ (20 ml). Purification by silica gel chromatography (1:5 EtOAc: hexane) gave 3d $(0.41 \mathrm{~g})$ in $60 \%$ yield as a colourless orange oil; b.p. $90{ }^{\circ} \mathrm{C}(5 \mathrm{mmbar}) ; v_{\max }\left(\mathrm{film} / \mathrm{cm}^{-1}\right) 3458,1687 ; \delta_{H}$ (400 MHz, CDCl $) 1.08$ (3H, t, J 7.3 Hz), $1.36(6 \mathrm{H}, \mathrm{s}), 2.27$ (2H, m), 3.99 (1H, bs), 6.39 $(1 \mathrm{H}, \mathrm{dd}, J$ 15.4, 2.2 and $1.5 \mathrm{~Hz}), 7.18(1 \mathrm{H}, \mathrm{m}) ; \delta_{C}\left(100 \mathrm{MHz}, \mathrm{CDCl}_{3}\right)$ 12.6, 26.2, 26.8, $75.6,121.9,152.8,202.9$.

(4E,6E)-2-Hydroxy-2-methyldeca-4,6-dien-3-one (3e). From (E)-hex-2-enal. Purification by silica gel chromatography (1:4 EtOAc: hexane) gave 3e $(0.41 \mathrm{~g})$ in $63 \%$ yield as a pale yellow oil; $v_{\max }\left(\mathrm{film} / \mathrm{cm}^{-1}\right) 3419,1635 ; \delta_{H}\left(400 \mathrm{MHz}, \mathrm{CDCl}_{3}\right) 0.94(3 \mathrm{H}, \mathrm{t}$, $J 7.3 \mathrm{~Hz}), 1.40(6 \mathrm{H}, \mathrm{s}), 1.49(2 \mathrm{H}, \mathrm{h}, J 7.4 \mathrm{~Hz}), 2.21(2 \mathrm{H}, \mathrm{q}, J 7.0 \mathrm{~Hz}), 4.07(1 \mathrm{H}, \mathrm{bs}), 6.26$ 
$(1 \mathrm{H}, \mathrm{dd}, J 15.1$ and $9.8 \mathrm{~Hz}), 6.36(1 \mathrm{H}, \mathrm{dt}, J 15.1$ and $6.8 \mathrm{~Hz}), 6.38(1 \mathrm{H}, \mathrm{d}, J 15.4 \mathrm{~Hz})$, $7.45(1 \mathrm{H}, \mathrm{dd}, J 15.4$ and $9.8 \mathrm{~Hz}) ; \delta_{C}\left(100 \mathrm{MHz}, \mathrm{CDCl}_{3}\right)$ 13.7, 21.8, 26.5, 35.2, 75.2, $119.8,128.8,146.1,147.84,175.9,202.79$.

(4E,6E)-2-Hydroxy-2,6-dimethylocta-4,6-dien-3-one (3f). From $0.80 \mathrm{ml}$ of 3hydroxy-3-methyl-2-butanone (7.58 mmol), $0.49 \mathrm{ml}(E)$-2-methylbut-2-enal (5.07 mmol), $1.78 \mathrm{ml}$ DIPA (12.64 mmol), and $7.90 \mathrm{ml}$ of ${ }^{\mathrm{n}} \mathrm{BuLi}(1.6 \mathrm{M}$ in hexanes, $12.64 \mathrm{mmol})$ in 25 $\mathrm{ml}$ of anhydrous THF. Purification by silica gel chromatography gave $\mathbf{3 f}$ in $15 \%$ yield. $v_{\max }\left(\mathrm{film} / \mathrm{cm}^{-1}\right) 3418,1686,1634 ; \delta_{H}\left(400 \mathrm{MHz}, \mathrm{CDCl}_{3}\right) 1.42(6 \mathrm{H}, \mathrm{s}), 1.82(3 \mathrm{H}, \mathrm{m}), 1.86$ (3H, d, J 7.5 Hz), $4.09(1 \mathrm{H}, \mathrm{s}), 6.16(1 \mathrm{H}, \mathrm{m}), 6.35(1 \mathrm{H}, \mathrm{d}, J 15.8 \mathrm{~Hz}), 7.50(1 \mathrm{H}, \mathrm{d}, J 15.8$ $\mathrm{Hz}) ; \delta_{C}\left(100 \mathrm{MHz}, \mathrm{CDCl}_{3}\right) 11.8,14.9,26.6,115.6,134.1,139.8,150.6,202.8$.

5-(4-Methoxyphenyl)-2,2-dimethylfuran-3(2H)-one (4b). ${ }^{3}$ Purification by silica gel (1:6, EtOAc: hexane) gave 4b in $74 \%$ yield; $v_{\max }\left(f i l m / \mathrm{cm}^{-1}\right) 1671,1610 ; \delta_{H}(400 \mathrm{MHz}$, $\left.\mathrm{CDCl}_{3}\right) 1.47(3 \mathrm{H}, \mathrm{s}), 3.87(3 \mathrm{H}, \mathrm{s}), 5.84(1 \mathrm{H}, \mathrm{s}), 6.95-6.99(2 \mathrm{H}, \mathrm{m}), 7.76-7.80(2 \mathrm{H}, \mathrm{m}) ; \delta_{C}$ $\left(100 \mathrm{MHz}, \mathrm{CDCl}_{3}\right)$ 23.2, 55.5, 88.8, 97.0, 114.2, 121.6, 129.1, 163.1, 183.2, 206.6; $\mathrm{m} / \mathrm{z}$ $219.1019\left(\mathrm{C}_{13} \mathrm{H}_{15} \mathrm{O}_{3}\right.$ requires 219.1021).

2,2-Dimethyl-5-phenylethyl-furan-3(2H)-one (4c). The reaction mixture was left to stir at $50{ }^{\circ} \mathrm{C}$ for seven days. Purification by silica gel chromatography (1:4, EtOAc:hexane) to give $4 \mathbf{c}$ as a pale yellow oil in $67 \%$ yield. $v_{\max }\left(\mathrm{film} / \mathrm{cm}^{-1}\right) 1700 ; \delta_{H}$ $\left(400 \mathrm{MHz}, \mathrm{CDCl}_{3}\right) 1.38(6 \mathrm{H}, \mathrm{s}), 2.85(2 \mathrm{H}, \mathrm{t}, J 7.6 \mathrm{~Hz}), 2.98(2 \mathrm{H}, \mathrm{t}, J 7.6 \mathrm{~Hz}), 5.32(1 \mathrm{H}$, s), 7.18-7.32 (5H, m); $\delta_{C}\left(100 \mathrm{MHz}, \mathrm{CDCl}_{3}\right) 22.8,32.1,32.5,88.7,101.6,126.6,128.3$, 128.6, 139.5, 176.0, 207.9; $\mathrm{m} / \mathrm{z} 217.1217\left(\mathrm{C}_{14} \mathrm{H}_{17} \mathrm{O}_{2}\right.$ requires 217.1229).

2,2-Dimethyl-5-ethyl-furan-3(2H)-one (4d). ${ }^{4}$ The reaction mixture was left to stir at $50{ }^{\circ} \mathrm{C}$ overnight. Purification by Kugelrohr distillation gave $\mathbf{4 d}$ as a colorless oil in $41 \%$ 
yield; bp $48-50{ }^{\circ} \mathrm{C} / 0.07 \mathrm{~mm} \mathrm{Hg} ; v_{\max }\left(\mathrm{film} / \mathrm{cm}^{-1}\right) 1700,1591 ; \delta_{H}\left(400 \mathrm{MHz}, \mathrm{CDCl}_{3}\right) 1.22$ $(3 \mathrm{H}, \mathrm{t}, J 7.6 \mathrm{~Hz}), 1.37(6 \mathrm{H}, \mathrm{s}), 2.49(2 \mathrm{H}, \mathrm{q}, J 7.6 \mathrm{~Hz}), 5.35(1 \mathrm{H}, \mathrm{s}) ; \delta_{C}\left(100 \mathrm{MHz}, \mathrm{CDCl}_{3}\right)$ 10.2, 22.8, 24.2, 88.5, 100.2, 193.0, 207.5; $\mathrm{m} / \mathrm{z}$ 141.0921 $\left(\mathrm{C}_{8} \mathrm{H}_{13} \mathrm{O}_{2}\right.$ requires 141.0916).

(E)-5-(But-2-en-2-yl)-2,2-dimethylfuran-3(2H)-one (4f). ${ }^{5}$ The reaction mixture was left to stir at $60^{\circ} \mathrm{C}$ for $30 \mathrm{~h}$. Purification by column chromatography (1:4, EtOAc:hexane) gave $4 \mathbf{f}$ as a pale yellow oil in $64 \%$ yield. $v_{\max }\left(\mathrm{film} / \mathrm{cm}^{-1}\right) 1704 ; \delta_{H}\left(400 \mathrm{MHz}, \mathrm{CDCl}_{3}\right)$ $1.36(6 \mathrm{H}, \mathrm{s}), 1.83(3 \mathrm{H}, \mathrm{dq}, J 7.1$ and $0.8 \mathrm{~Hz}), 1.87(3 \mathrm{H}, \mathrm{dq}, J 1.3$ and $0.8 \mathrm{~Hz}), 5.45(1 \mathrm{H}$, s), $6.68(1 \mathrm{H}, \mathrm{qq}, J 7.1$ and $1.3 \mathrm{~Hz}) ; \delta_{C}\left(100 \mathrm{MHz}, \mathrm{CDCl}_{3}\right) 13.9,14.2,23.1,88.1,98.5$, 133.1, 136.9, 184.5, 207.3; m/z 167.1078 $\left(\mathrm{C}_{10} \mathrm{H}_{15} \mathrm{O}_{2}\right.$ requires 167.1072).

Ethyl-syn-2,3-dihydroxy-2-methyl-5-phenylpentenoate (6). ${ }^{6} \mathrm{From}^{\mathrm{OsO}} \mathrm{O}_{4}(20 \mathrm{~mol} \%)$, NMO (120 mol\%) and 5 in THF. The reaction mixture was left to stir at rt overnight. Purification by silica gel chromatography (1:3, EtOAc:hexane) gave $\mathbf{6}$ as a pale orange oil in $100 \%$ yield, with syn/anti $=88 / 12 . v_{\max }\left(\mathrm{film} / \mathrm{cm}^{-1}\right) 3419,1645 ; \delta_{H}(400 \mathrm{MHz}$, $\left.\left.\mathrm{CDCl}_{3}\right)\right) 1.31(3 \mathrm{H}, \mathrm{t}, J \quad 7.1 \mathrm{~Hz}), 1.34(3 \mathrm{H}, \mathrm{s}), 1.76(1 \mathrm{H}, \mathrm{dddd}, J$ 14.1, 10.8, 9.6 and 5.0 Hz), $1.93(1 \mathrm{H}$, dddd, $J$ 14.1, 9.6, 7.3 and $2.2 \mathrm{~Hz}), 2.05(1 \mathrm{H}, \mathrm{d}, J 9.4 \mathrm{~Hz}), 2.69(1 \mathrm{H}$, ddd, $J$ 14.0, 9.6 and 7.3 Hz), 2.95 (1H, ddd, $J$ 14.0, 9.8 and $4.8 \mathrm{~Hz}), 3.44(1 \mathrm{H}, \mathrm{bs}), 3.75(\mathrm{td}, J$ 10.8, 2.2), $4.27(2 \mathrm{H}, \mathrm{qd}, J$ 7.1, $2.0 \mathrm{~Hz}), 7.18-7.33(5 \mathrm{H}, \mathrm{m}) ; \delta_{C}\left(100 \mathrm{MHz}, \mathrm{CDCl}_{3}\right) 14.1$, $21.7,32.1,32.3,62.3,74.9,77.1,126.0,128.4,128.5,141.9,176.2 ; \mathrm{m} / \mathrm{z} 253.1426$ $\left(\mathrm{C}_{14} \mathrm{H}_{21} \mathrm{O}_{4}\right.$ requires 253.1440$)$.

Ethyl-2,2,4-trimethyl-5-phenylethyl-1,3-dioxolane-4-carboxylate (7). From 2,2dimethoxypropane $(1 \mathrm{ml} / \mathrm{mmol})$, pTSA $(5 \mathrm{~mol} \%)$ and 6 in acetone. The reaction mixture was left to stir at rt overnight. Purification by silica gel chromatography (1:8, EtOAc:hexane) gave 7 as a colourless oil in $94 \%$ yield, $d e=76 \% . v_{\max }\left(\mathrm{film} / \mathrm{cm}^{-1}\right) 1645$; 
$\delta_{H}\left(400 \mathrm{MHz}, \mathrm{CDCl}_{3}\right) 1.25(3 \mathrm{H}, \mathrm{t}, J \quad 7.1 \mathrm{~Hz}), 1.34(3 \mathrm{H}, \mathrm{s}), 1.41(3 \mathrm{H}, \mathrm{s}), 1.91(2 \mathrm{H}, \mathrm{m})$, $2.69(1 \mathrm{H}, \mathrm{dt}, J 14.0$ and $8.0 \mathrm{~Hz}), 2.89(1 \mathrm{H}, \mathrm{ddd}, J$ 14.0, 7.3 and $7.0 \mathrm{~Hz}), 4.18(1 \mathrm{H}, \mathrm{dd}, J$ 7.3 and $7.0 \mathrm{~Hz}), 4.19\left(2 \mathrm{H}, \mathrm{q}, J\right.$ 7.1 Hz), 7.18-7.33 (5H, m); $\delta_{C}\left(100 \mathrm{MHz}, \mathrm{CDCl}_{3}\right)$ 14.1, $19.9,25.5,28.3,31.5,32.8,61.3,79.3,82.4,109.0,126.0,128.4,128.5,141.5,173.2$ 293.1742 $\left(\mathrm{C}_{14} \mathrm{H}_{25} \mathrm{O}_{4}\right.$ requires 293.1753).

Syn-5,6-dihydroxy-5-methyl-8-phenyl-oct-2-en-4-one (10). From 9 (120 mg) and 2:3 TFA/ $\mathrm{H}_{2} \mathrm{O}(5 \mathrm{ml})$. The reaction was stirred at $\mathrm{rt}$ for $3 \mathrm{~h}$. Purification by silica gel chromarography (1:3 EtOAc:hexane) gave $\mathbf{1 0}$ as a colourless oil in $92 \%$ yield, de $>99 \%$. $v_{\max }\left(\mathrm{film} / \mathrm{cm}^{-1}\right) 3442,1642 ; \delta_{H}\left(400 \mathrm{MHz}, \mathrm{CDCl}_{3}\right) 1.27(3 \mathrm{H}, \mathrm{s}), 1.82(1 \mathrm{H}, \mathrm{dddd}, J$ 14.6, 10.3, 9.8 and $5.3 \mathrm{~Hz}), 1.95(1 \mathrm{H}, \mathrm{m}), 1.96(3 \mathrm{H}, \mathrm{dd}, J 6.8$ and $1.5 \mathrm{~Hz}), 2.69(1 \mathrm{H}$, ddd, $J$ 14.1, 9.3 and $7.5 \mathrm{~Hz}), 2.95(1 \mathrm{H}$, ddd, $J 14.3,9.6$ and $5.3 \mathrm{~Hz}), 3.82(1 \mathrm{H}, \mathrm{dd}, J 10.6$ and 2.0 $\mathrm{Hz}), 6.49(1 \mathrm{H}, \mathrm{dd}, J 15.0$ and $1.5 \mathrm{~Hz}), 7.18-7.31(5 \mathrm{H}, \mathrm{m}), 7.25(1 \mathrm{H}, \mathrm{dd}, J 17.0$ and 10.2 $\mathrm{Hz}) ; \delta_{C}\left(100 \mathrm{MHz}, \mathrm{CDCl}_{3}\right)$ 18.7, 21.3, 32.0, 32.4, 74.0, 80.0, 124.0, 125.9, 128.3, 128.4, 141.8, 146.8, 200.9; $m / z 248.1409\left(\mathrm{C}_{15} \mathrm{H}_{20} \mathrm{O}_{3}\right.$ requires 248.1412).

1-(2,2,4-trimethyl-5-phenylethyl-1,3-dioxolane-4-yl)-prop-2-en-1-one (12). From vinylmagnesium bromide ( 3 equiv.) and $\mathbf{8}$ in THF. Purification by silica gel chromatography (1:5, EtOAc:hexane) gave $\mathbf{1 2}$ as a pale yellow oil in $80 \%$ yield, de $>99 \%$. $v_{\max }$ $\left(\right.$ film $\left./ \mathrm{cm}^{-1}\right) 1644 ; \quad \delta_{H}\left(400 \mathrm{MHz}, \mathrm{CDCl}_{3}\right) 1.27(3 \mathrm{H}, \mathrm{s}), 1.38(3 \mathrm{H}, \mathrm{s}), 1.52(3 \mathrm{H}, \mathrm{s}), 1.87$ $(1 \mathrm{H}$, ddd, $J$ 8.8, 7.6 and $6.6 \mathrm{~Hz}), 1.89(1 \mathrm{H}$, ddd, $J$ 8.3, 7.6 and $6.6 \mathrm{~Hz}), 2.68(1 \mathrm{H}$, ddd, $J$ 14.4, 8.8 and $8.0 \mathrm{~Hz}), 2.87(1 \mathrm{H}$, ddd, $J 14.4,8.0$ and $7.5 \mathrm{~Hz}), 4.45(1 \mathrm{H}, \mathrm{dd}, J \quad 6.8$ and 5.5 $\mathrm{Hz}), 5.77(1 \mathrm{H}, \mathrm{dd}, J 10.3$ and $2.0 \mathrm{~Hz}), 6.42(1 \mathrm{H}, \mathrm{dd}, J 17.2$ and $2.0 \mathrm{~Hz}), 7.07(1 \mathrm{H}, \mathrm{dd}, J$ 17.0 and $10.6 \mathrm{~Hz}), 7.17-7.31(5 \mathrm{H}, \mathrm{m}) ; \delta_{C}\left(100 \mathrm{MHz}, \mathrm{CDCl}_{3}\right)$ 19.1, 26.0, 28.5, 31.6, 32.9, 
$78.2,86.7,108.7,125.9,128.4,128.5,130.2,130.3,141.5,201.2 ; \mathrm{m} / z 275.1637$ $\left(\mathrm{C}_{17} \mathrm{H}_{23} \mathrm{O}_{3}\right.$ requires 275.1647).

Syn-4,5-dihydroxy-4-methyl-7-phenylhept-1-en-3-one (13). From 12 (100 mg) and 2:3 TFA $/ \mathrm{H}_{2} \mathrm{O}(5 \mathrm{ml})$. The reaction was stirred at $\mathrm{rt}$ for $3 \mathrm{~h}$. Purification by silica gel chromarography (1:3 EtOAc:hexane) gave $\mathbf{1 3}$ in 90\% yield as a colourless oil, de >99\%. $v_{\max }\left(\mathrm{film} / \mathrm{cm}^{-1}\right) 3442,1788,1694 ; \delta_{H}\left(400 \mathrm{MHz}, \mathrm{CDCl}_{3}\right) 1.29(3 \mathrm{H}, \mathrm{s}), 1.81(1 \mathrm{H}$, dddd, $J$ 14.3, 10.6, 9.2 and $5.1 \mathrm{~Hz}), 1.95$ (1H, dddd, $J$ 14.0, 9.6, 7.2 and $2.4 \mathrm{~Hz}), 2.69(1 \mathrm{H}, \mathrm{ddd}, J$ 14.0 9.3 and 7.5 Hz), $2.93(1 \mathrm{H}$, ddd, $J$ 14.1, 9.6 and $5.1 \mathrm{~Hz}), 3.80(1 \mathrm{H}, \mathrm{dd}, J 10.6$ and 2.0 Hz), $4.05(1 \mathrm{H}, \mathrm{bs}), 5.84(1 \mathrm{H}, \mathrm{dd}, J 10.2$ and $1.7 \mathrm{~Hz}), 6.54(1 \mathrm{H}, \mathrm{dd}, J 17.0$ and $1.7 \mathrm{~Hz})$, $6.77(1 \mathrm{H}, \mathrm{dd}, J 17.0$ and $10.2 \mathrm{~Hz}), 7.18-7.31(5 \mathrm{H}, \mathrm{m}) ; \delta_{C}\left(100 \mathrm{MHz}, \mathrm{CDCl}_{3}\right) 21.2,31.9$, $32.3,74.3,80.4,125.9,128.4,128.5,129.1,131.4,141.7,201.7 ; m / z 234.1249\left(\mathrm{C}_{14} \mathrm{H}_{18} \mathrm{O}_{3}\right.$ requires 234.1256).

2-(1-Hydroxy-3-phenylpropyl)-2-methyl-furan-3(2H)-one (14). From 11.35 mg of $\mathrm{PdCl}_{2}(0.064 \mathrm{mmol}), 6.3 \mathrm{mg}$ of $\mathrm{CuCl}(0.064 \mathrm{mmol}), 9.0 \mathrm{mg}$ of $\mathrm{Na}_{2} \mathrm{HPO}_{4}(0.064 \mathrm{mmol})$ and $0.150 \mathrm{~g}$ of $13(0.64 \mathrm{mmol})$. The reaction mixture was stirred at $50^{\circ} \mathrm{C}$ overnight. Purification by silica gel chromatography (1:4; EtOAc:hexane) gave $\mathbf{1 4}$ in $50 \%$ yield as a white solid. $v_{\max }\left(\mathrm{film} / \mathrm{cm}^{-1}\right) 3419,1651 ; \delta_{H}\left(400 \mathrm{MHz}, \mathrm{CDCl}_{3}\right) 1.42(3 \mathrm{H}, \mathrm{s}), 1.68(1 \mathrm{H}$, dddd, $J$ 14.0, 10.6, 9.6 and $4.8 \mathrm{~Hz}), 2.05(1 \mathrm{H}$, dddd, $J$ 13.9, 9.6, 7.3 and $2.2 \mathrm{~Hz}), 2.65$ (1H, ddd, $J 14.09 .3$ and $7.5 \mathrm{~Hz}), 2.93(1 \mathrm{H}$, ddd, $J$ 14.1, 9.8 and $2.2 \mathrm{~Hz}), 3.76(1 \mathrm{H}, \mathrm{dd}, J$ 10.8 and $2.0 \mathrm{~Hz}), 5.66(1 \mathrm{H}, \mathrm{d}, J 2.3 \mathrm{~Hz}), 7.17-7.31(5 \mathrm{H}, \mathrm{m}), 8.23(1 \mathrm{H}, \mathrm{d}, J 2.3 \mathrm{~Hz}) ; \delta_{C}$ $\left(100 \mathrm{MHz}, \mathrm{CDCl}_{3}\right)$ 18.4, 32.0, 32.2, 73.4, 90.5, 106.8, 126.0, 128.4, 128.5, 141.6, 177.6, 207.2; $\mathrm{m} / \mathrm{z} 232.1092\left(\mathrm{C}_{14} \mathrm{H}_{16} \mathrm{O}_{3}\right.$ requires 232.1099). 


\section{X-ray data for 2-(1-hydroxy-3-phenylpropyl)-2,5-dimethyl-furan-3(2H)-one 11}

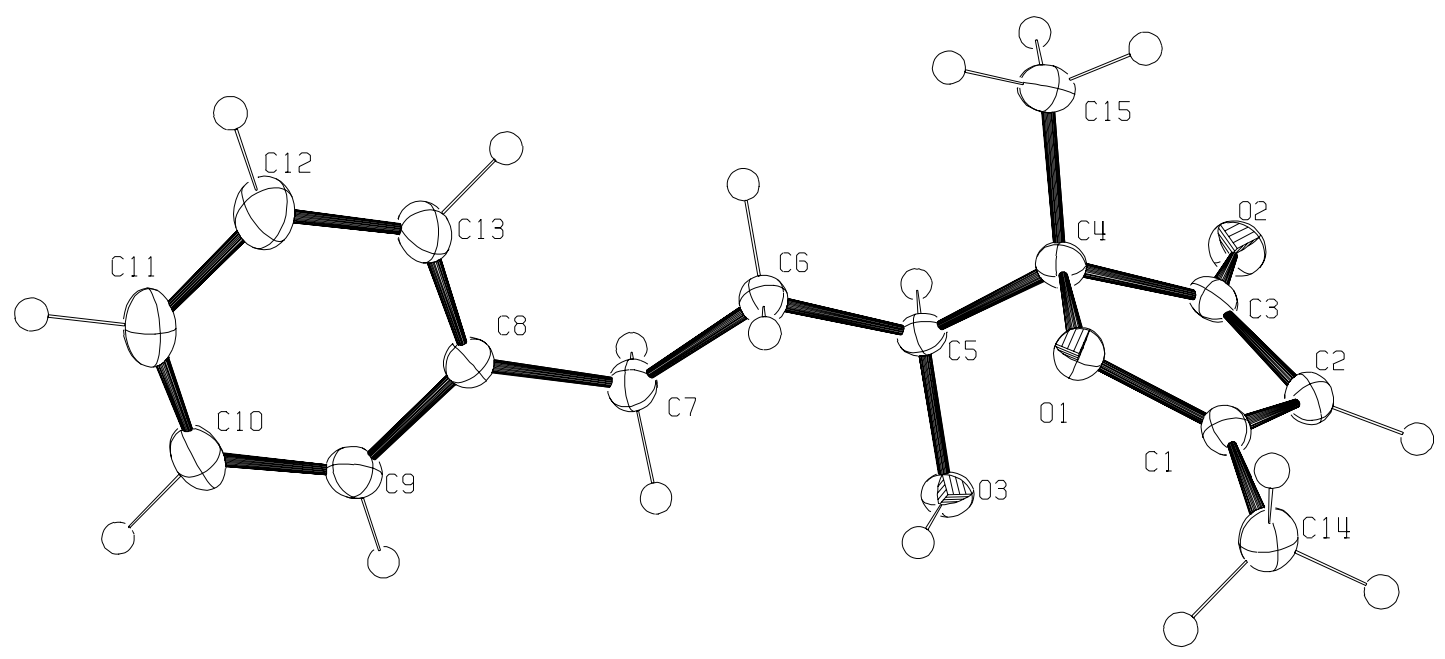

Crystals were grown by recrystallization from ethyl acetate. A single crystal having dimensions of approximately $0.08 \times 0.10 \times 0.40 \mathrm{~mm}$ was mounted on a glass fibre using perfluoropolyether oil and cooled rapidly to $150 \mathrm{~K}$ in a stream of cold $\mathrm{N}_{2}$ using an Oxford Cryosystems CRYOSTREAM unit. Diffraction data were measured using an EnrafNonius KappaCCD diffractometer (graphite-monochromated $\mathrm{MoK}_{\alpha}$ radiation, $\lambda=$ $0.71073 \AA$ ). Intensity data were processed using the DENZO-SMN package ${ }^{6}$.

The structure was solved in the space group $P \overline{1}$ using the direct-methods program $\mathrm{SIR}^{7}{ }^{7}$, which located all non-hydrogen atoms. Subsequent full-matrix least-squares refinement was carried out using the CRYSTALS program suite ${ }^{8}$. Coordinates and anisotropic thermal parameters of all non-hydrogen atoms were refined. The $\mathrm{OH}$ hydrogen atoms was located in a difference Fourier map and its coordinates and isotropic thermal parameter subsequently refined. Other hydrogen atoms were positioned geometrically after each cycle of refinement. A 3-term Chebychev polynomial weighting 
scheme was applied. Refinement converged satisfactorily to give $\mathrm{R}=0.0369, \mathrm{wR}=$ 0.0462 .

Attached is a thermal ellipsoid plot (ORTEP- $\left.3^{9}\right)$ at $40 \%$ probability. A summary of crystallographic data is given below, as are full lists of atomic coordinates, anisotropic thermal parameters and those bond lengths and angles not concerning geometricallypositioned $\mathrm{H}$ atoms.

\section{Comment:}

The $\mathrm{OH}$ group forms a hydrogen bond to the carbonyl $\mathrm{O}$ atom of a neighbouring molecule $\left(\mathrm{O}(3) \cdots \mathrm{O}(2)^{\prime} 2.7874(14) \AA\right.$, symmetry operator $\left.1+x, y, z\right)$, leading to the formation of infinite chains running parallel to the crystallographic $a$ axis. 
Table 1: Crystal data and refinement details

\begin{tabular}{|c|c|}
\hline Crystal identification & ARC1181 \\
\hline Chemical formula & $\mathrm{C}_{15} \mathrm{H}_{18} \mathrm{O}_{3}$ \\
\hline Formula weight & 246.31 \\
\hline Temperature $(\mathrm{K})$ & 150 \\
\hline Wavelength $(\AA)$ & 0.71073 \\
\hline Crystal system & Triclinic \\
\hline Space group & $P \overline{1}$ \\
\hline$a(\AA)$ & $5.8675(2)$ \\
\hline$b(\AA)$ & $10.7248(3)$ \\
\hline$c(\AA)$ & $11.1452(4)$ \\
\hline$\alpha\left(^{\circ}\right)$ & $77.1515(10)$ \\
\hline$\beta\left(^{\circ}\right)$ & $77.8504(10)$ \\
\hline$\gamma\left({ }^{\circ}\right)$ & $85.1546(15)$ \\
\hline Cell volume $\left(\AA^{3}\right)$ & $667.94(4)$ \\
\hline Z & 2 \\
\hline Calculated density $\left(\mathrm{Mg} / \mathrm{m}^{3}\right)$ & 1.225 \\
\hline Absorption coefficient $\left(\mathrm{mm}^{-1}\right)$ & 0.084 \\
\hline $\mathrm{F}_{000}$ & 264 \\
\hline Crystal size $(\mathrm{mm})$ & $0.08 \times 0.10 \times 0.40$ \\
\hline Description of crystal & Colourless prism \\
\hline Absorption correction & Semi-empirical from equivalent reflections \\
\hline Transmission coefficients (min,max) & $0.97,0.99$ \\
\hline$\theta$ range for data collection $\left(^{\circ}\right)$ & $5.0 \leq \theta \leq 27.5$ \\
\hline Index ranges & $-7 \leq h \leq 7,-13 \leq k \leq 13,0 \leq I \leq 14$ \\
\hline Reflections measured & 9853 \\
\hline Unique reflections & 3033 \\
\hline $\mathrm{R}_{\text {int }}$ & 0.034 \\
\hline Observed reflections $(I>3 \sigma(I))$ & 2088 \\
\hline Refinement method & Full-matrix least-squares on $F$ \\
\hline Parameters refined & 167 \\
\hline Weighting scheme & Chebychev 3-term polynomial \\
\hline Goodness of fit & 0.9802 \\
\hline $\mathrm{R}$ & 0.0369 \\
\hline$w R$ & 0.0462 \\
\hline Residual electron density $(\min , \max )\left(\mathrm{e}^{-3}\right)$ & $-0.17,0.24$ \\
\hline
\end{tabular}


Table 2: Atomic coordinates and equivalent isotropic thermal parameters $\left(\AA^{2}\right)$ of non-hydrogen atoms

\begin{tabular}{|c|c|c|c|c|}
\hline Atom & $\mathrm{X}$ & $\mathrm{y}$ & $\mathrm{Z}$ & $\mathrm{U}_{\text {equiv }}$ \\
\hline & & & & \\
\hline $\mathrm{O}(1)$ & $0.09224(16)$ & $0.17447(9)$ & $0.25037(9)$ & 0.0224 \\
\hline $\mathrm{C}(1)$ & $0.0367(2)$ & $0.05361(13)$ & $0.31285(13)$ & 0.0235 \\
\hline $\mathrm{C}(2)$ & $-0.1830(2)$ & $0.04282(13)$ & $0.38114(14)$ & 0.0257 \\
\hline $\mathrm{C}(3)$ & $-0.2898(2)$ & $0.16849(14)$ & $0.36923(13)$ & 0.0234 \\
\hline $\mathrm{C}(4)$ & $-0.1097(2)$ & $0.25916(13)$ & $0.28072(13)$ & 0.0215 \\
\hline $\mathrm{C}(5)$ & $-0.0368(2)$ & $0.35396(13)$ & $0.34973(12)$ & 0.0211 \\
\hline $\mathrm{C}(6)$ & $0.1383(2)$ & $0.44824(13)$ & $0.26348(13)$ & 0.0234 \\
\hline $\mathrm{C}(7)$ & $0.2361(3)$ & $0.53109(14)$ & $0.33442(13)$ & 0.0265 \\
\hline $\mathrm{C}(8)$ & $0.4202(2)$ & $0.62186(13)$ & $0.25460(13)$ & 0.0240 \\
\hline $\mathrm{C}(9)$ & $0.5766(3)$ & $0.66755(14)$ & $0.31159(15)$ & 0.0303 \\
\hline $\mathrm{C}(11)$ & $0.7471(3)$ & $0.75134(16)$ & $0.24254(18)$ & 0.0369 \\
\hline $\mathrm{C}(12)$ & $0.7646(3)$ & $0.79196(17)$ & $0.11435(18)$ & 0.0407 \\
\hline $\mathrm{C}(13)$ & $0.6113(3)$ & $0.74794(19)$ & $0.05601(17)$ & 0.0444 \\
\hline $\mathrm{C}(14)$ & $0.4403(3)$ & $0.66371(17)$ & $0.12564(15)$ & 0.0356 \\
\hline $\mathrm{O}(2)$ & $0.2268(3)$ & $-0.04462(14)$ & $0.29502(15)$ & 0.0306 \\
\hline $\mathrm{C}(15)$ & $-0.48591(16)$ & $0.20540(10)$ & $0.41663(10)$ & 0.0308 \\
\hline $\mathrm{O}(3)$ & $-0.1943(3)$ & $0.32113(15)$ & $0.16065(14)$ & 0.0295 \\
\hline & $0.04455(17)$ & $0.28468(9)$ & $0.45809(9)$ & 0.0236 \\
\hline
\end{tabular}

Table 3: Atomic coordinates and isotropic thermal parameters $\left(\AA^{2}\right)$ of hydrogen atoms

\begin{tabular}{|c|c|c|c|c|}
\hline Atom & $\mathrm{X}$ & $\mathrm{y}$ & $\mathrm{Z}$ & $\mathrm{U}_{\text {iso }}$ \\
\hline & & & & \\
\hline $\mathrm{H}(1)$ & $0.190(4)$ & $0.261(2)$ & $0.430(2)$ & $0.057(6)$ \\
\hline $\mathrm{H}(21)$ & -0.2571 & -0.0382 & 0.4309 & 0.0310 \\
\hline $\mathrm{H}(51)$ & -0.1763 & 0.4083 & 0.3779 & 0.0249 \\
\hline $\mathrm{H}(61)$ & 0.0589 & 0.5054 & 0.1994 & 0.0280 \\
\hline $\mathrm{H}(62)$ & 0.2706 & 0.3989 & 0.2204 & 0.0280 \\
\hline$H(71)$ & 0.1036 & 0.5831 & 0.3738 & 0.0317 \\
\hline$H(72)$ & 0.3067 & 0.4730 & 0.4016 & 0.0317 \\
\hline$H(91)$ & 0.5655 & 0.6392 & 0.4043 & 0.0378 \\
\hline$H(101)$ & 0.8576 & 0.7825 & 0.2855 & 0.0463 \\
\hline$H(111)$ & 0.8871 & 0.8526 & 0.0643 & 0.0477 \\
\hline$H(121)$ & 0.6232 & 0.7767 & -0.0367 & 0.0518 \\
\hline$H(131)$ & 0.3302 & 0.6328 & 0.0822 & 0.0429 \\
\hline$H(141)$ & 0.1714 & -0.1303 & 0.3440 & 0.0366 \\
\hline$H(142)$ & 0.3644 & -0.0228 & 0.3252 & 0.0366 \\
\hline$H(143)$ & 0.2729 & -0.0470 & 0.2039 & 0.0366 \\
\hline$H(151)$ & -0.3329 & 0.3795 & 0.1803 & 0.0359 \\
\hline$H(152)$ & -0.0667 & 0.3715 & 0.1003 & 0.0359 \\
\hline$H(153)$ & -0.2392 & 0.2532 & 0.1223 & 0.0359 \\
\hline
\end{tabular}


Table 4: Anisotropic thermal parameters $\left(\AA^{2}\right)$

\begin{tabular}{|c|c|c|c|c|c|c|}
\hline Atom & $\mathrm{U}_{11}$ & $\mathrm{U}_{22}$ & $\mathrm{U}_{33}$ & $\mathrm{U}_{23}$ & $\mathrm{U}_{13}$ & $\mathrm{U}_{12}$ \\
\hline & & & & & & \\
\hline $\mathrm{O}(1)$ & $0.0198(5)$ & $0.0220(5)$ & $0.0242(5)$ & $-0.0054(4)$ & $-0.0016(4)$ & $0.0009(4)$ \\
\hline $\mathrm{C}(1)$ & $0.0250(7)$ & $0.0226(6)$ & $0.0249(7)$ & $-0.0068(5)$ & $-0.0075(5)$ & $-0.0010(5)$ \\
\hline $\mathrm{C}(2)$ & $0.0233(6)$ & $0.0246(7)$ & $0.0295(7)$ & $-0.0050(6)$ & $-0.0061(5)$ & $-0.0027(5)$ \\
\hline $\mathrm{C}(3)$ & $0.0192(6)$ & $0.0291(7)$ & $0.0233(7)$ & $-0.0056(5)$ & $-0.0067(5)$ & $-0.0024(5)$ \\
\hline $\mathrm{C}(4)$ & $0.0171(6)$ & $0.0238(6)$ & $0.0230(7)$ & $-0.0054(5)$ & $-0.0034(5)$ & $0.0020(5)$ \\
\hline $\mathrm{C}(5)$ & $0.0194(6)$ & $0.0219(6)$ & $0.0209(6)$ & $-0.0033(5)$ & $-0.0043(5)$ & $0.0027(5)$ \\
\hline $\mathrm{C}(6)$ & $0.0234(6)$ & $0.0237(7)$ & $0.0228(7)$ & $-0.0048(5)$ & $-0.0041(5)$ & $-0.0014(5)$ \\
\hline $\mathrm{C}(7)$ & $0.0305(7)$ & $0.0270(7)$ & $0.0218(7)$ & $-0.0046(5)$ & $-0.0039(5)$ & $-0.0045(6)$ \\
\hline $\mathrm{C}(8)$ & $0.0246(7)$ & $0.0213(6)$ & $0.0266(7)$ & $-0.0065(5)$ & $-0.0052(5)$ & $0.0014(5)$ \\
\hline $\mathrm{C}(9)$ & $0.0329(8)$ & $0.0263(7)$ & $0.0354(8)$ & $-0.0093(6)$ & $-0.0132(6)$ & $0.0015(6)$ \\
\hline $\mathrm{C}(10)$ & $0.0290(8)$ & $0.0342(8)$ & $0.0527(10)$ & $-0.0156(7)$ & $-0.0117(7)$ & $-0.0039(6)$ \\
\hline $\mathrm{C}(11)$ & $0.0314(8)$ & $0.0382(9)$ & $0.0498(10)$ & $-0.0104(7)$ & $0.0034(7)$ & $-0.0110(7)$ \\
\hline $\mathrm{C}(12)$ & $0.0450(10)$ & $0.0539(11)$ & $0.0306(8)$ & $-0.0030(8)$ & $0.0008(7)$ & $-0.0187(8)$ \\
\hline $\mathrm{C}(13)$ & $0.0360(8)$ & $0.0444(9)$ & $0.0268(8)$ & $-0.0047(7)$ & $-0.0052(6)$ & $-0.0145(7)$ \\
\hline $\mathrm{C}(14)$ & $0.0274(7)$ & $0.0262(7)$ & $0.0380(8)$ & $-0.0106(6)$ & $-0.0036(6)$ & $0.0029(6)$ \\
\hline $\mathrm{O}(2)$ & $0.0163(5)$ & $0.0377(6)$ & $0.0363(6)$ & $-0.0070(5)$ & $-0.0018(4)$ & $0.0007(4)$ \\
\hline $\mathrm{C}(15)$ & $0.0282(7)$ & $0.0327(7)$ & $0.0288(8)$ & $-0.0033(6)$ & $-0.0114(6)$ & $-0.0008(6)$ \\
\hline $\mathrm{O}(3)$ & $0.0214(5)$ & $0.0273(5)$ & $0.0206(5)$ & $-0.0029(4)$ & $-0.0039(4)$ & $0.0007(4)$ \\
\hline
\end{tabular}

Table 5: Bond lengths ( $\AA$ )

\begin{tabular}{|c|l|c|c|}
\hline $\mathrm{O}(1)-\mathrm{C}(1)$ & $1.3586(17)$ & $\mathrm{C}(5)-\mathrm{O}(3)$ & $1.4222(16)$ \\
\hline $\mathrm{O}(1)-\mathrm{C}(4)$ & $1.4592(16)$ & $\mathrm{C}(6)-\mathrm{C}(7)$ & $1.5274(19)$ \\
\hline $\mathrm{C}(1)-\mathrm{C}(2)$ & $1.352(2)$ & $\mathrm{C}(7)-\mathrm{C}(8)$ & $1.515(2)$ \\
\hline $\mathrm{C}(1)-\mathrm{C}(14)$ & $1.4816(19)$ & $\mathrm{C}(8)-\mathrm{C}(9)$ & $1.395(2)$ \\
\hline $\mathrm{C}(2)-\mathrm{C}(3)$ & $1.428(2)$ & $\mathrm{C}(8)-\mathrm{C}(13)$ & $1.390(2)$ \\
\hline $\mathrm{C}(3)-\mathrm{C}(4)$ & $1.5310(19)$ & $\mathrm{C}(9)-\mathrm{C}(10)$ & $1.386(2)$ \\
\hline $\mathrm{C}(3)-\mathrm{O}(2)$ & $1.2328(17)$ & $\mathrm{C}(10)-\mathrm{C}(11)$ & $1.382(3)$ \\
\hline $\mathrm{C}(4)-\mathrm{C}(5)$ & $1.5387(19)$ & $\mathrm{C}(11)-\mathrm{C}(12)$ & $1.382(3)$ \\
\hline $\mathrm{C}(4)-\mathrm{C}(15)$ & $1.5192(19)$ & $\mathrm{C}(12)-\mathrm{C}(13)$ & $1.392(2)$ \\
\hline $\mathrm{C}(5)-\mathrm{C}(6)$ & $1.5265(19)$ & $\mathrm{O}(3)-\mathrm{H}(1)$ & $0.88(2)$ \\
\hline
\end{tabular}

Note - geometrically-positioned $\mathrm{H}$ atoms have been excluded

Table 6: Bond angles $\left({ }^{\circ}\right)$

\begin{tabular}{|c|c|c|c|}
\hline $\mathrm{C}(1)-\mathrm{O}(1)-\mathrm{C}(4)$ & $107.75(10)$ & $\mathrm{C}(4)-\mathrm{C}(5)-\mathrm{C}(6)$ & $112.22(11)$ \\
\hline $\mathrm{O}(1)-\mathrm{C}(1)-\mathrm{C}(2)$ & $114.66(12)$ & $\mathrm{C}(4)-\mathrm{C}(5)-\mathrm{O}(3)$ & $109.31(10)$ \\
\hline $\mathrm{O}(1)-\mathrm{C}(1)-\mathrm{C}(14)$ & $114.72(12)$ & $\mathrm{C}(6)-\mathrm{C}(5)-\mathrm{O}(3)$ & $113.05(11)$ \\
\hline $\mathrm{C}(2)-\mathrm{C}(1)-\mathrm{C}(14)$ & $130.63(13)$ & $\mathrm{C}(5)-\mathrm{C}(6)-\mathrm{C}(7)$ & $112.62(11)$ \\
\hline $\mathrm{C}(1)-\mathrm{C}(2)-\mathrm{C}(3)$ & $107.40(12)$ & $\mathrm{C}(6)-\mathrm{C}(7)-\mathrm{C}(8)$ & $114.98(12)$ \\
\hline $\mathrm{C}(2)-\mathrm{C}(3)-\mathrm{C}(4)$ & $106.59(11)$ & $\mathrm{C}(7)-\mathrm{C}(8)-\mathrm{C}(9)$ & $119.38(13)$ \\
\hline $\mathrm{C}(2)-\mathrm{C}(3)-\mathrm{O}(2)$ & $130.43(13)$ & $\mathrm{C}(7)-\mathrm{C}(8)-\mathrm{C}(13)$ & $122.95(13)$ \\
\hline $\mathrm{C}(4)-\mathrm{C}(3)-\mathrm{O}(2)$ & $122.99(13)$ & $\mathrm{C}(9)-\mathrm{C}(8)-\mathrm{C}(13)$ & $117.66(13)$ \\
\hline $\mathrm{O}(1)-\mathrm{C}(4)-\mathrm{C}(3)$ & $103.56(10)$ & $\mathrm{C}(8)-\mathrm{C}(9)-\mathrm{C}(10)$ & $121.45(15)$ \\
\hline $\mathrm{O}(1)-\mathrm{C}(4)-\mathrm{C}(5)$ & $107.60(10)$ & $\mathrm{C}(9)-\mathrm{C}(10)-\mathrm{C}(11)$ & $120.12(14)$ \\
\hline $\mathrm{C}(3)-\mathrm{C}(4)-\mathrm{C}(5)$ & $110.72(11)$ & $\mathrm{C}(10)-\mathrm{C}(11)-\mathrm{C}(12)$ & $119.37(15)$ \\
\hline
\end{tabular}




\begin{tabular}{|c|c|c|c|}
\hline $\mathrm{O}(1)-\mathrm{C}(4)-\mathrm{C}(15)$ & $108.76(11)$ & $\mathrm{C}(11)-\mathrm{C}(12)-\mathrm{C}(13)$ & $120.37(16)$ \\
\hline $\mathrm{C}(3)-\mathrm{C}(4)-\mathrm{C}(15)$ & $110.91(11)$ & $\mathrm{C}(8)-\mathrm{C}(13)-\mathrm{C}(12)$ & $121.03(15)$ \\
\hline $\mathrm{C}(5)-\mathrm{C}(4)-\mathrm{C}(15)$ & $114.63(11)$ & $\mathrm{C}(5)-\mathrm{O}(3)-\mathrm{H}(1)$ & $104.8(16)$ \\
\hline
\end{tabular}

Note - geometrically-positioned $\mathrm{H}$ atoms have been excluded 


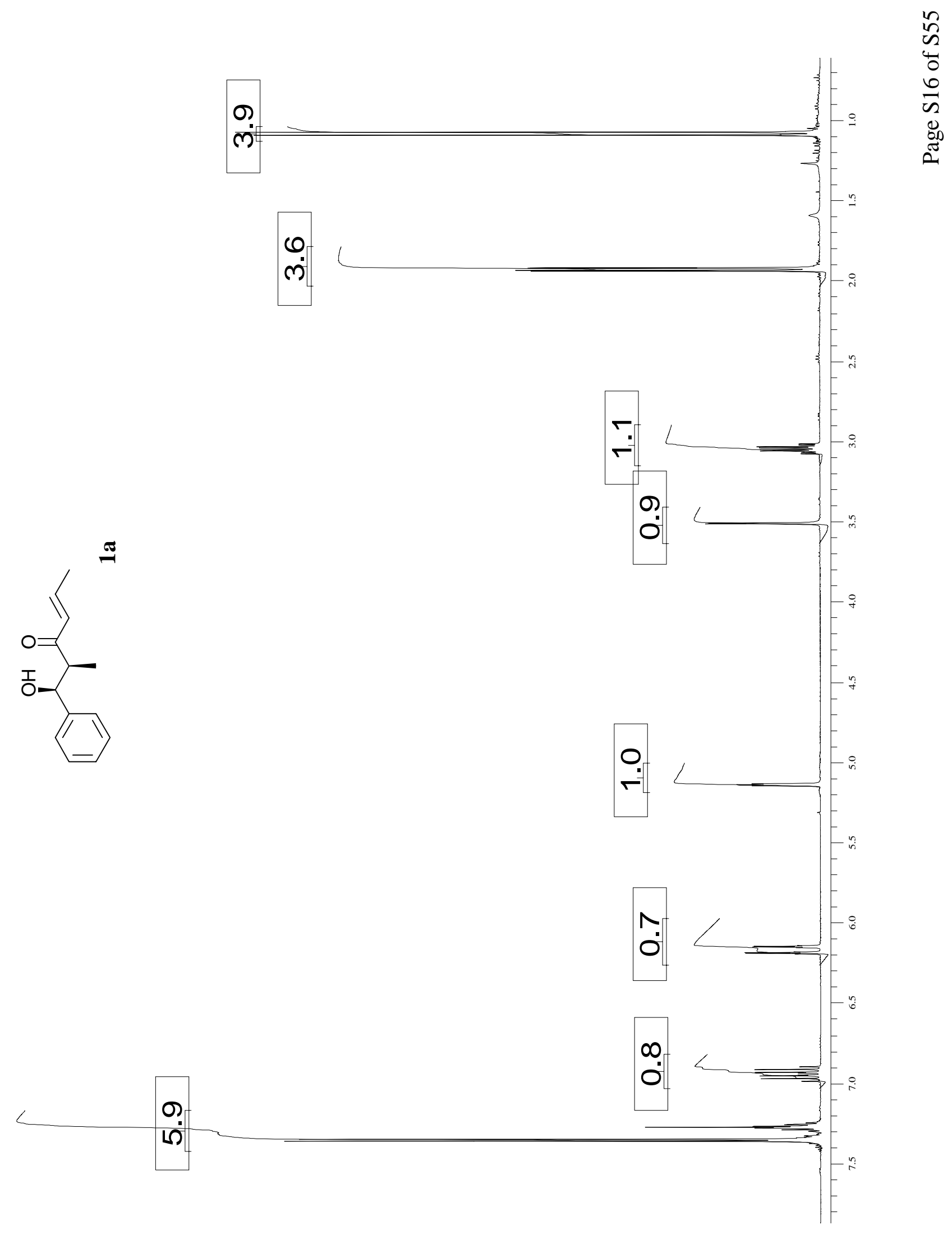




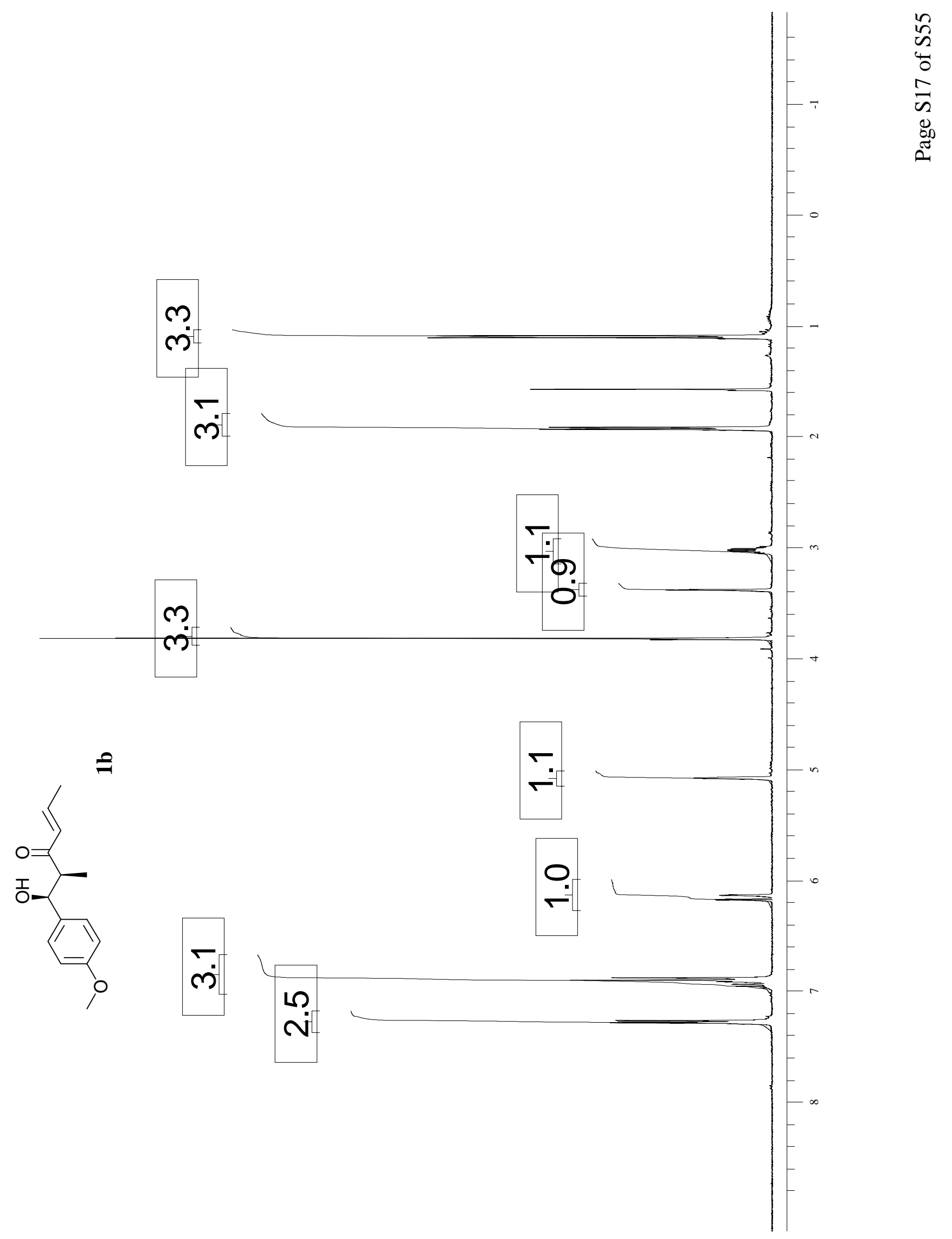




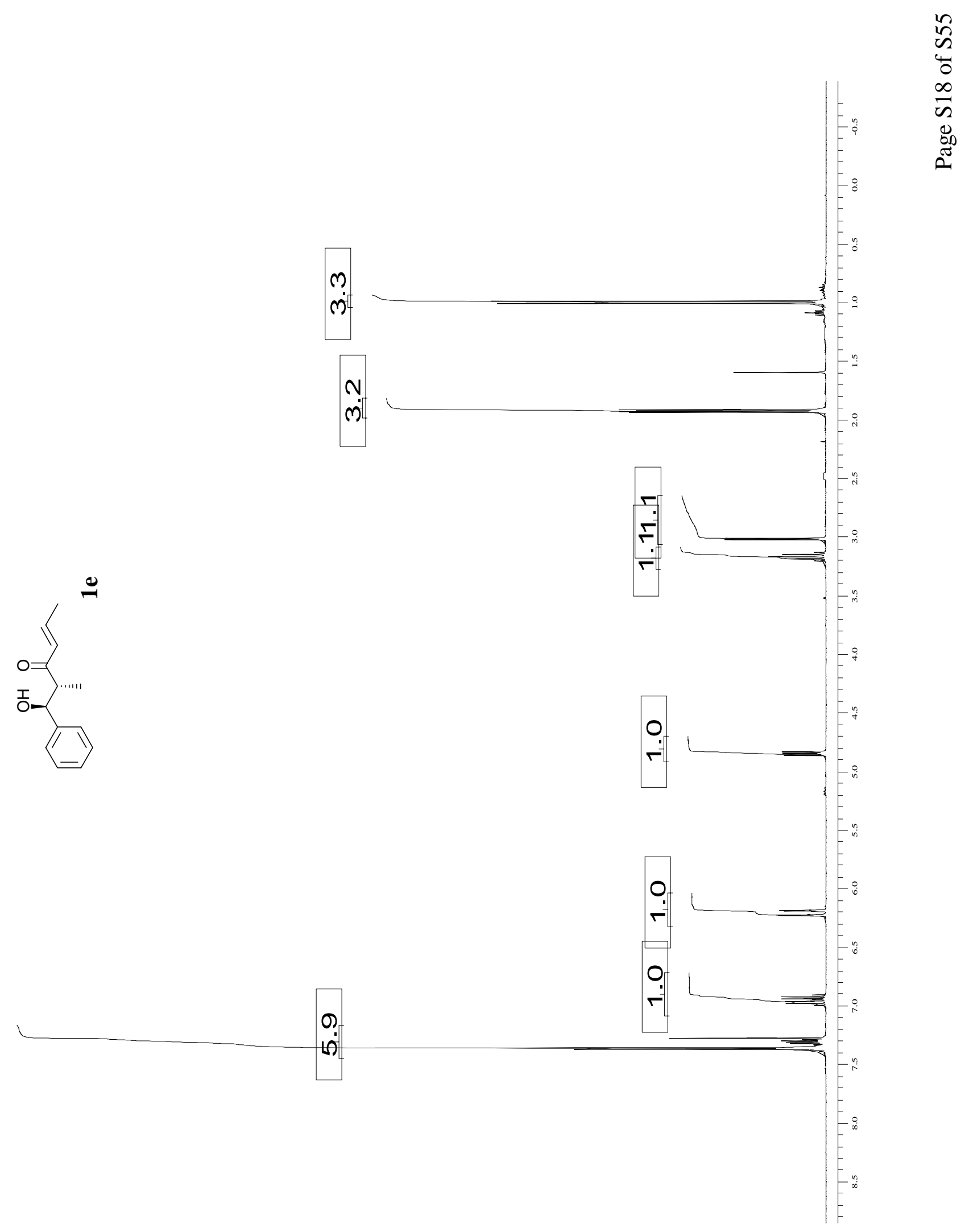




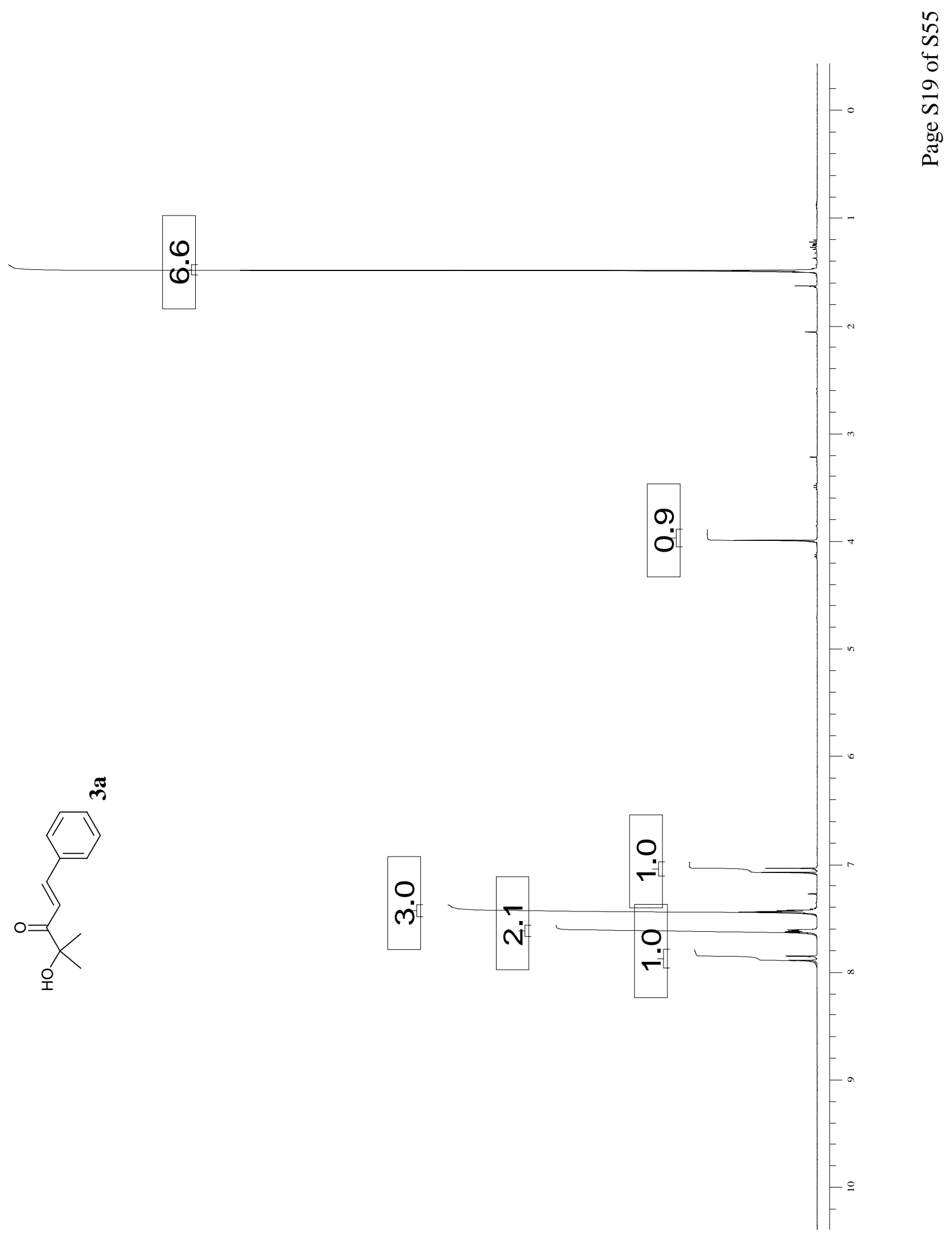




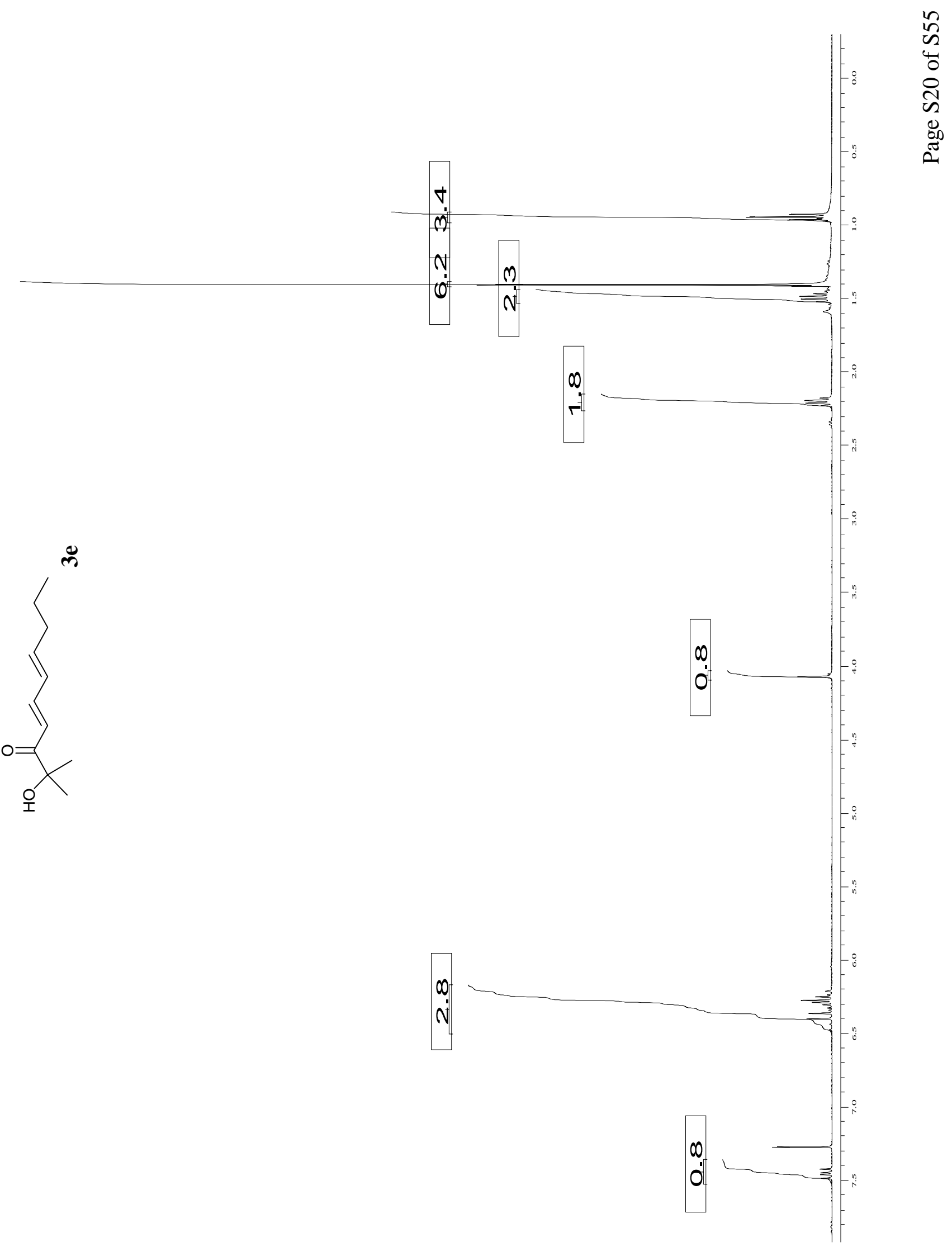




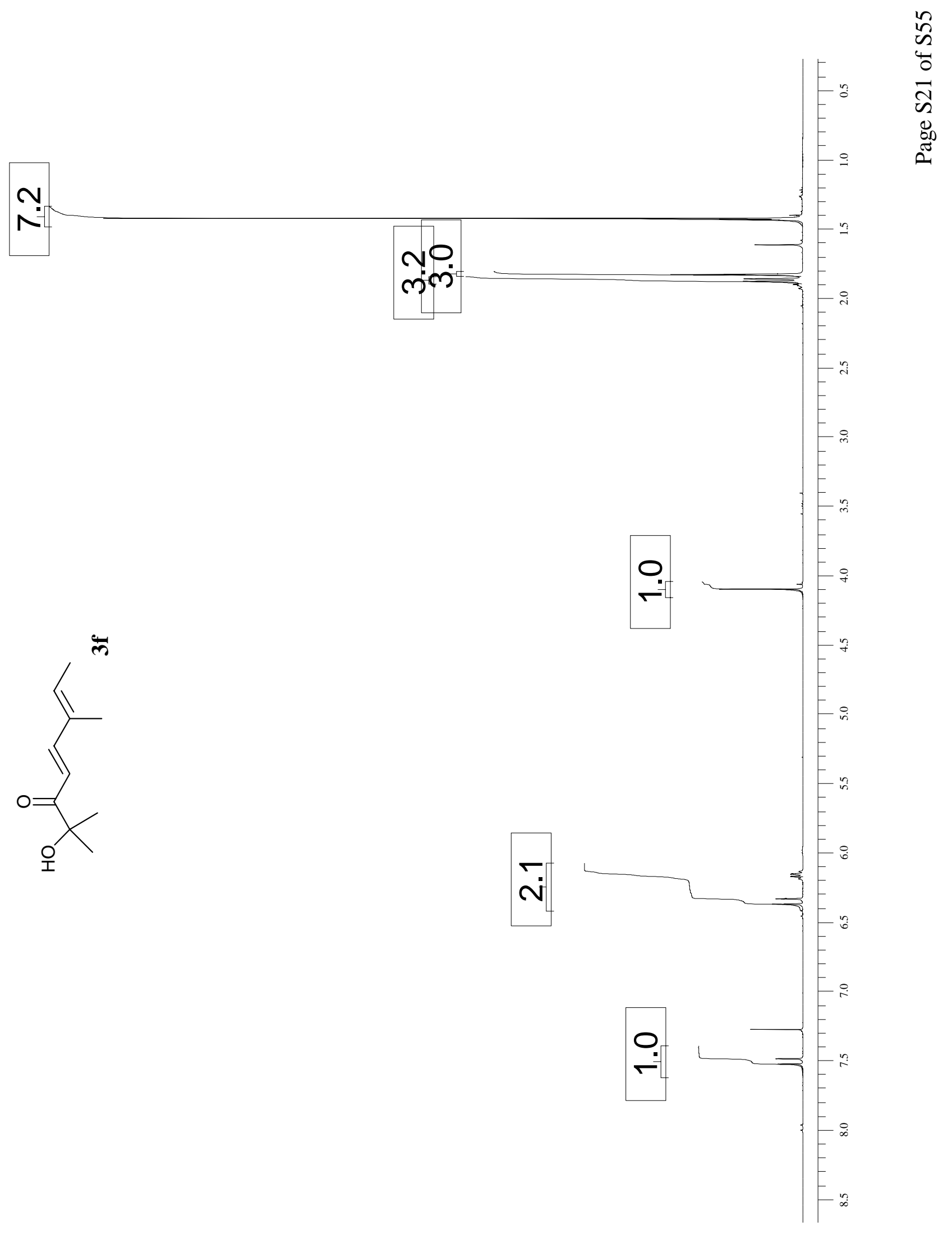




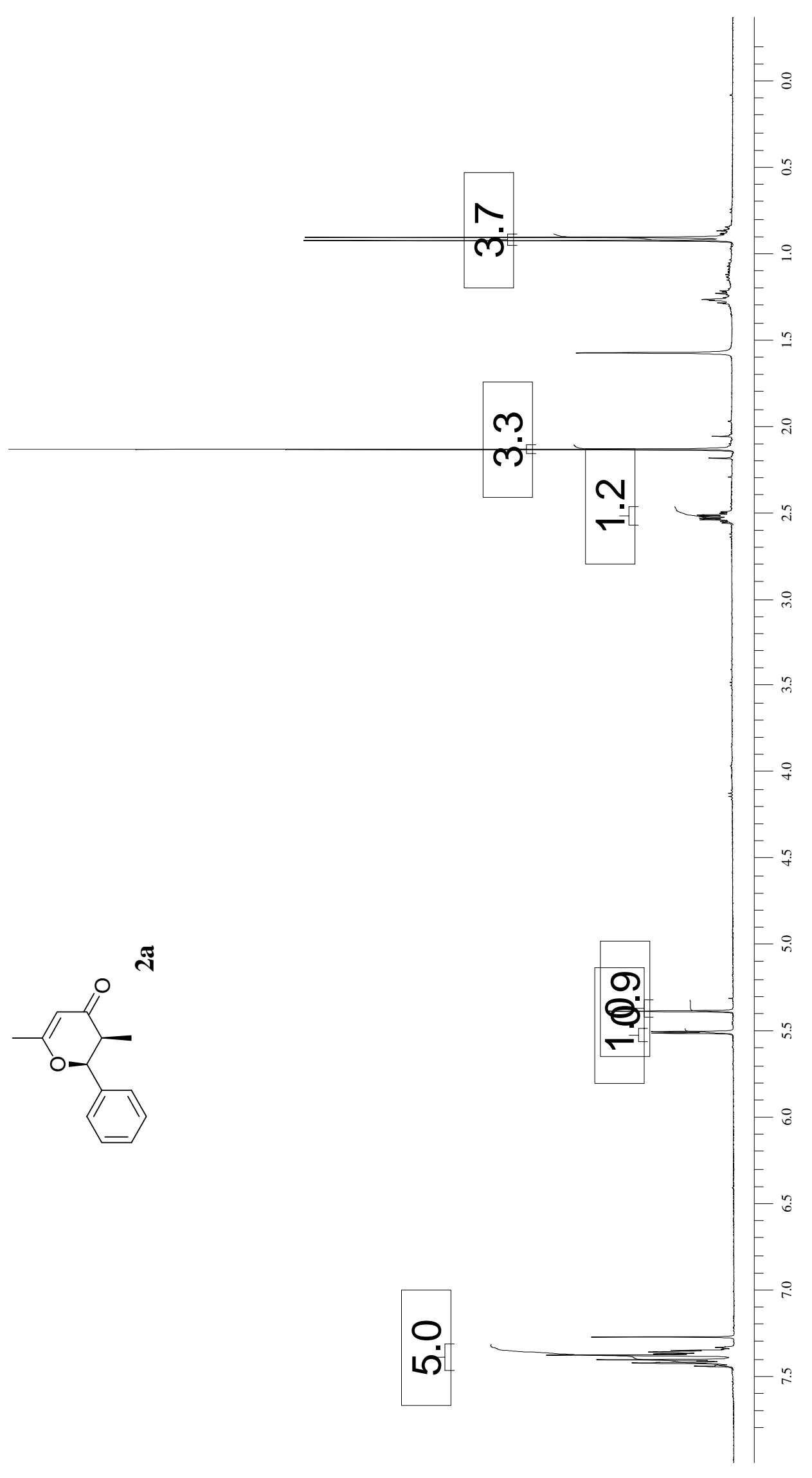

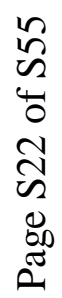




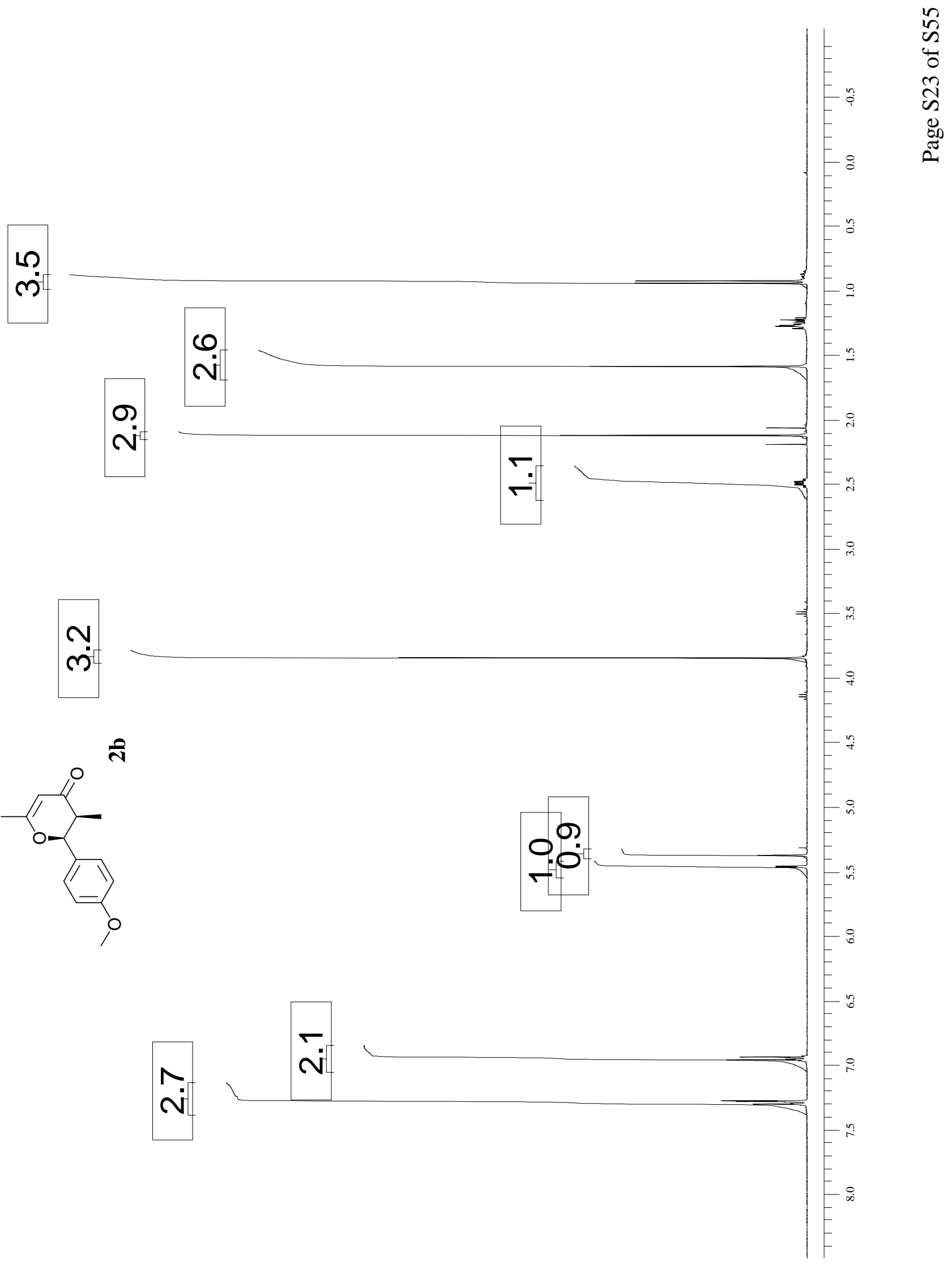




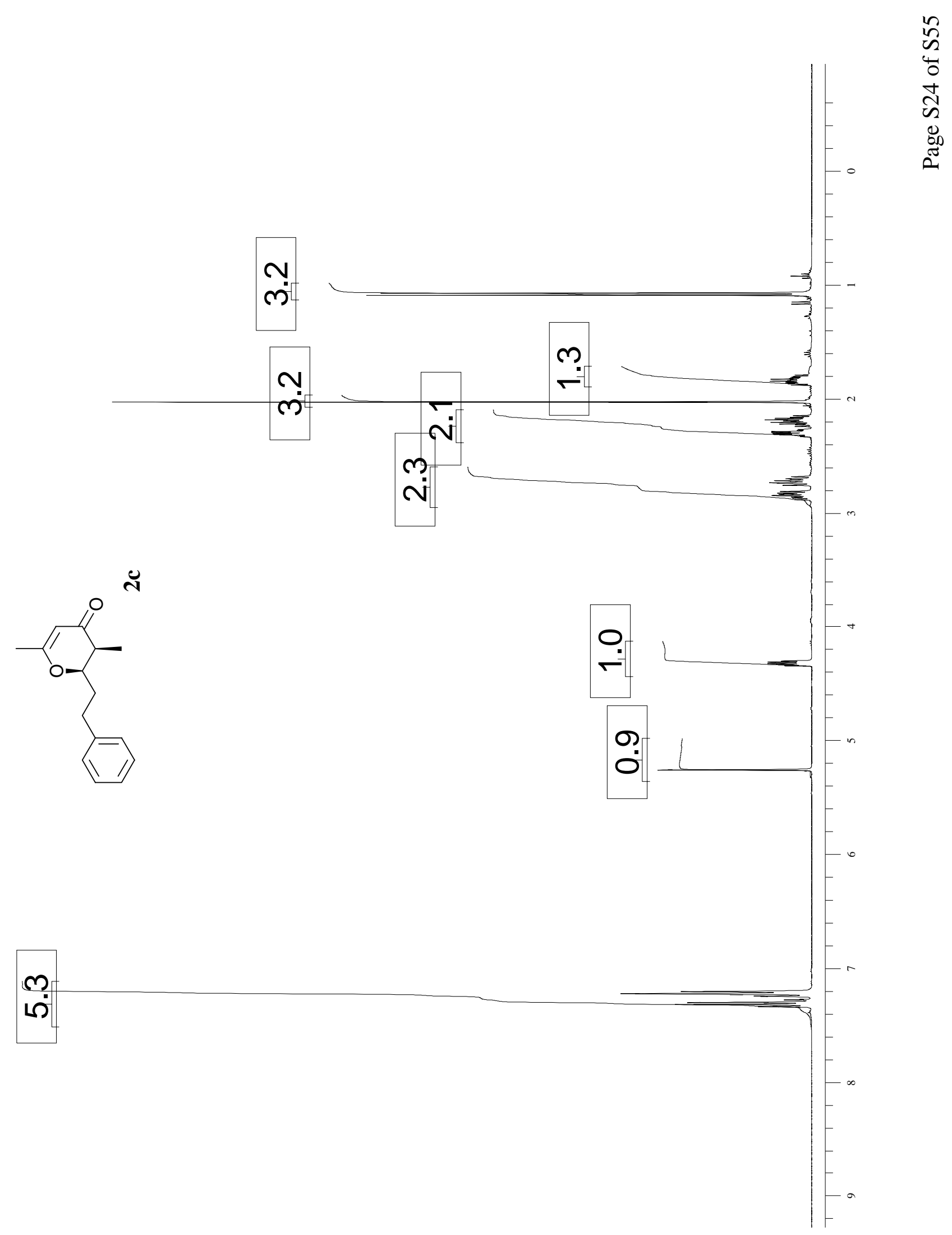




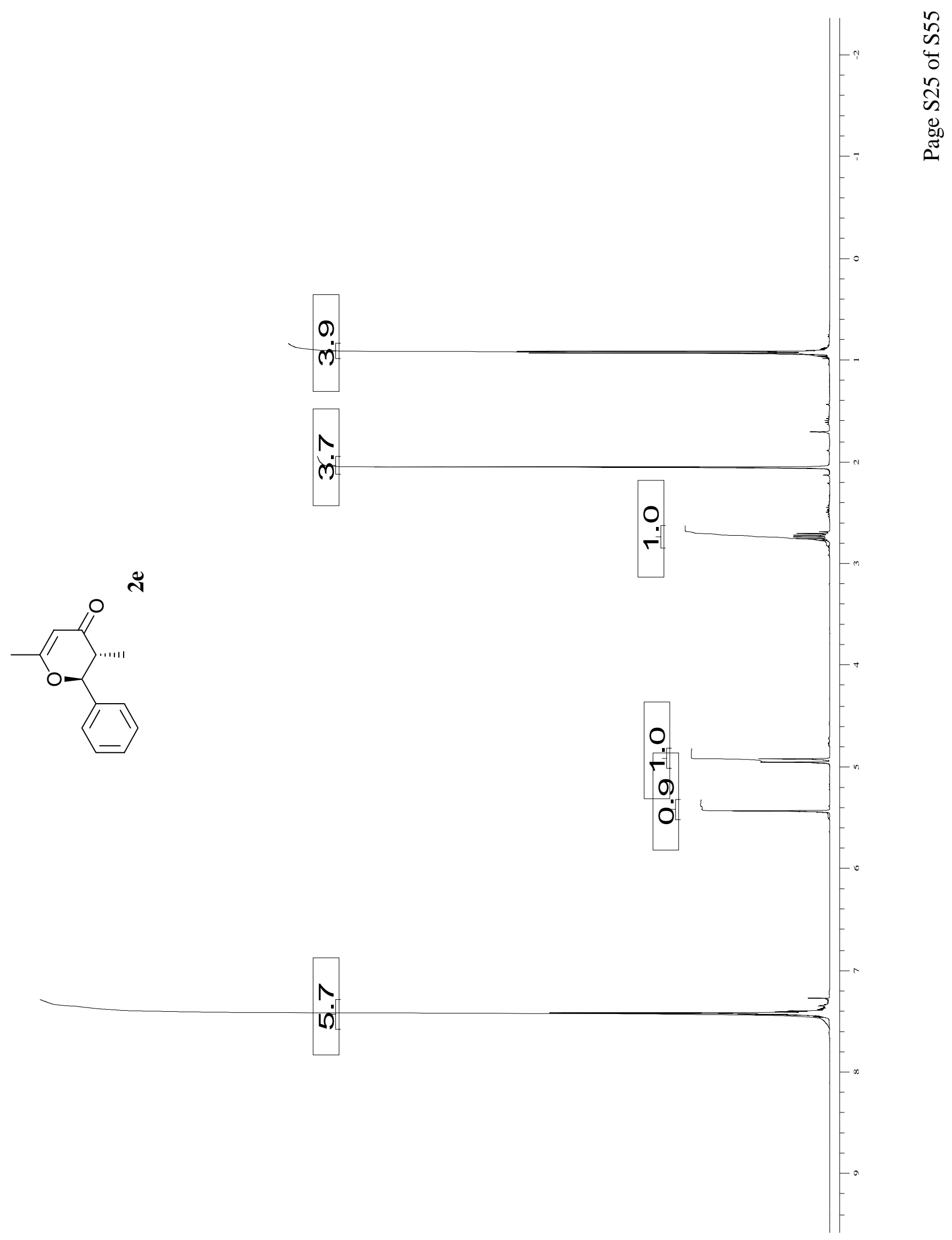




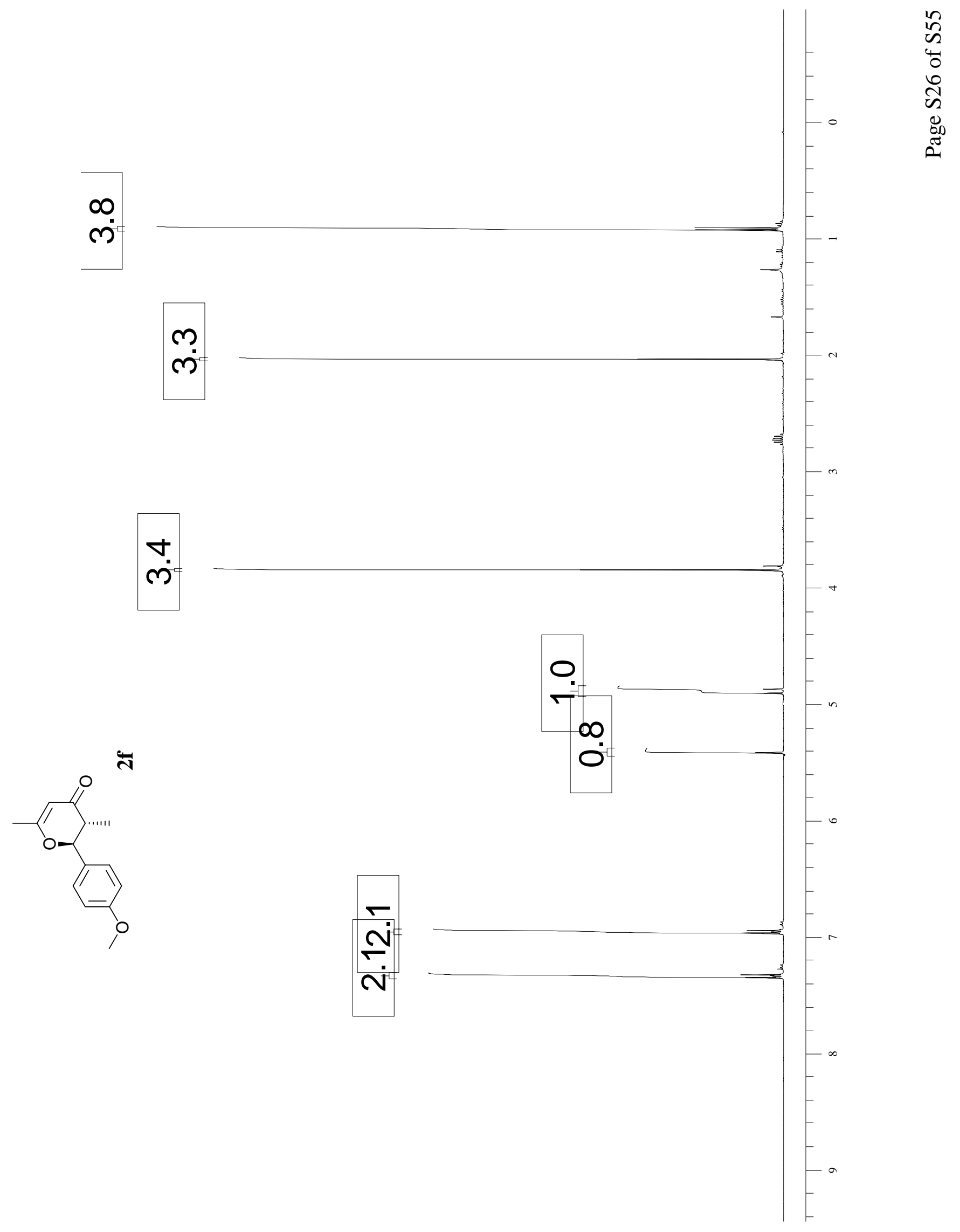




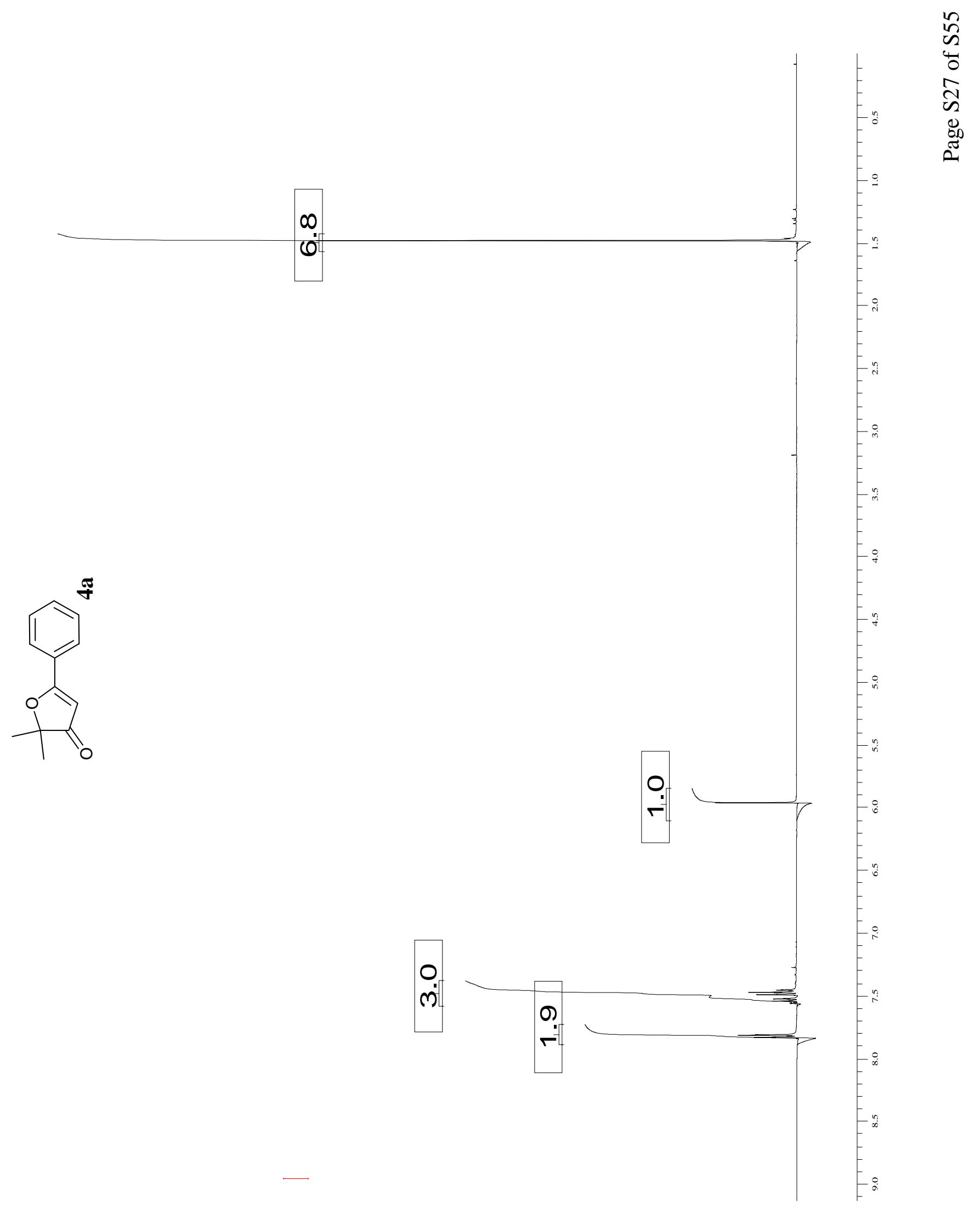




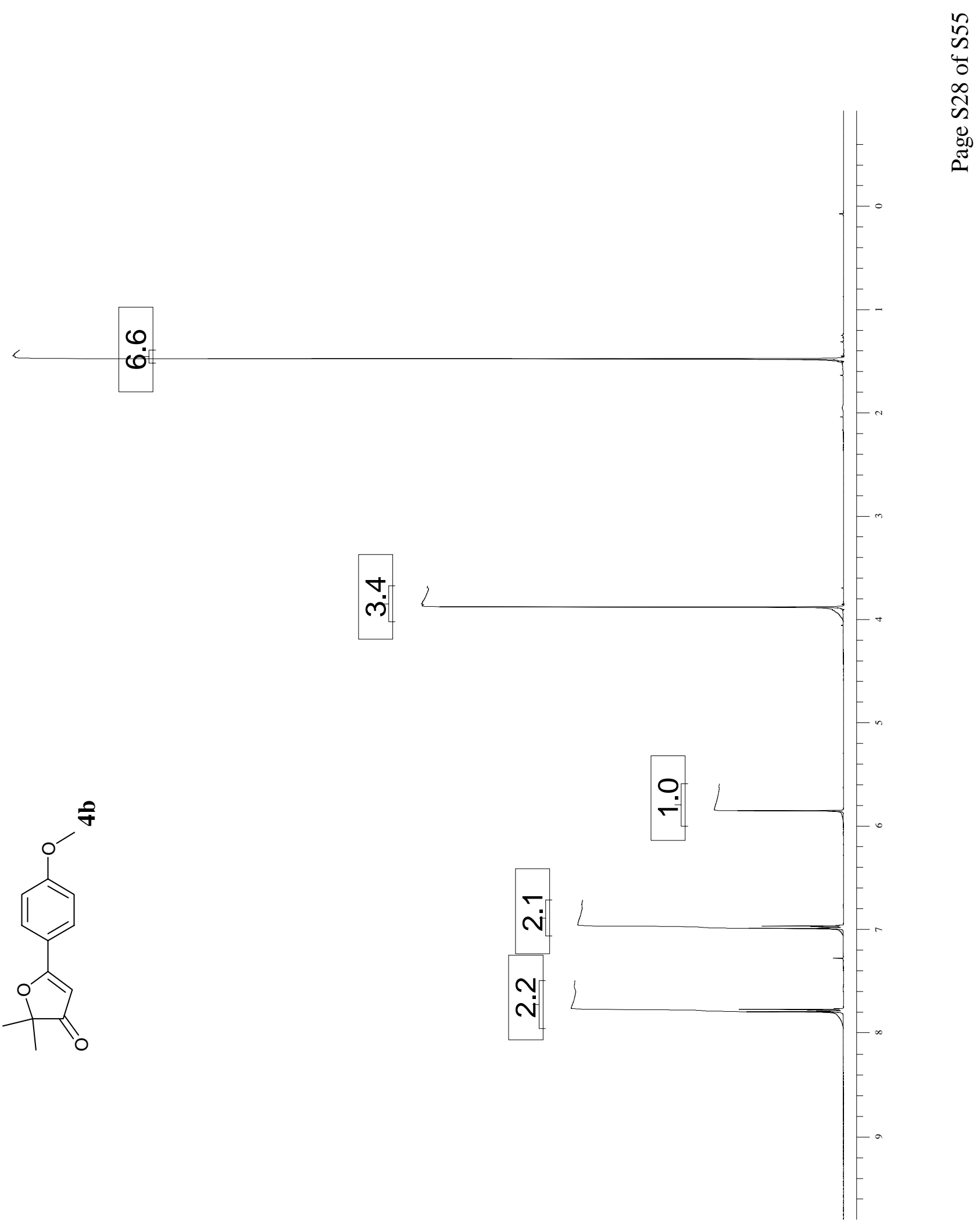




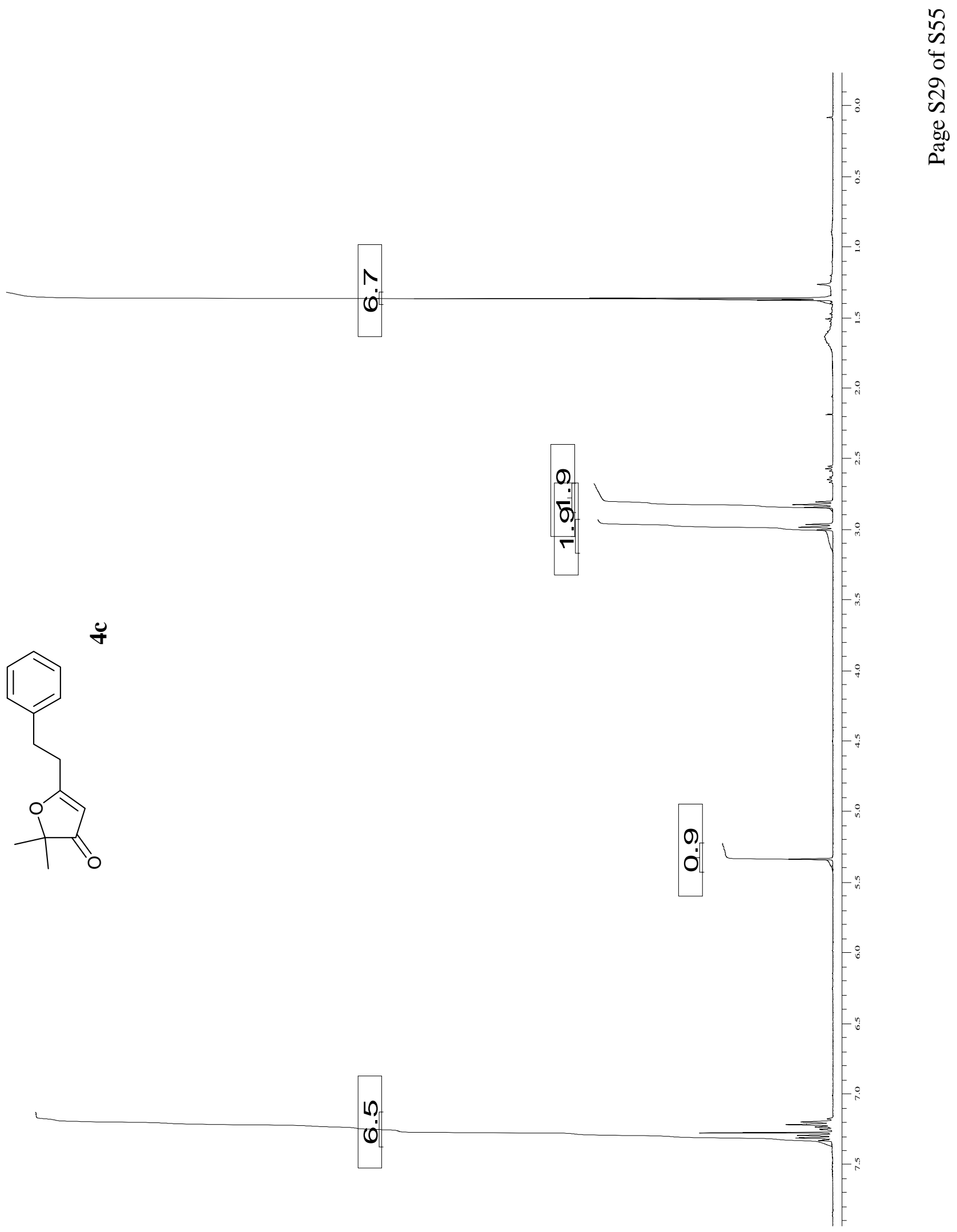




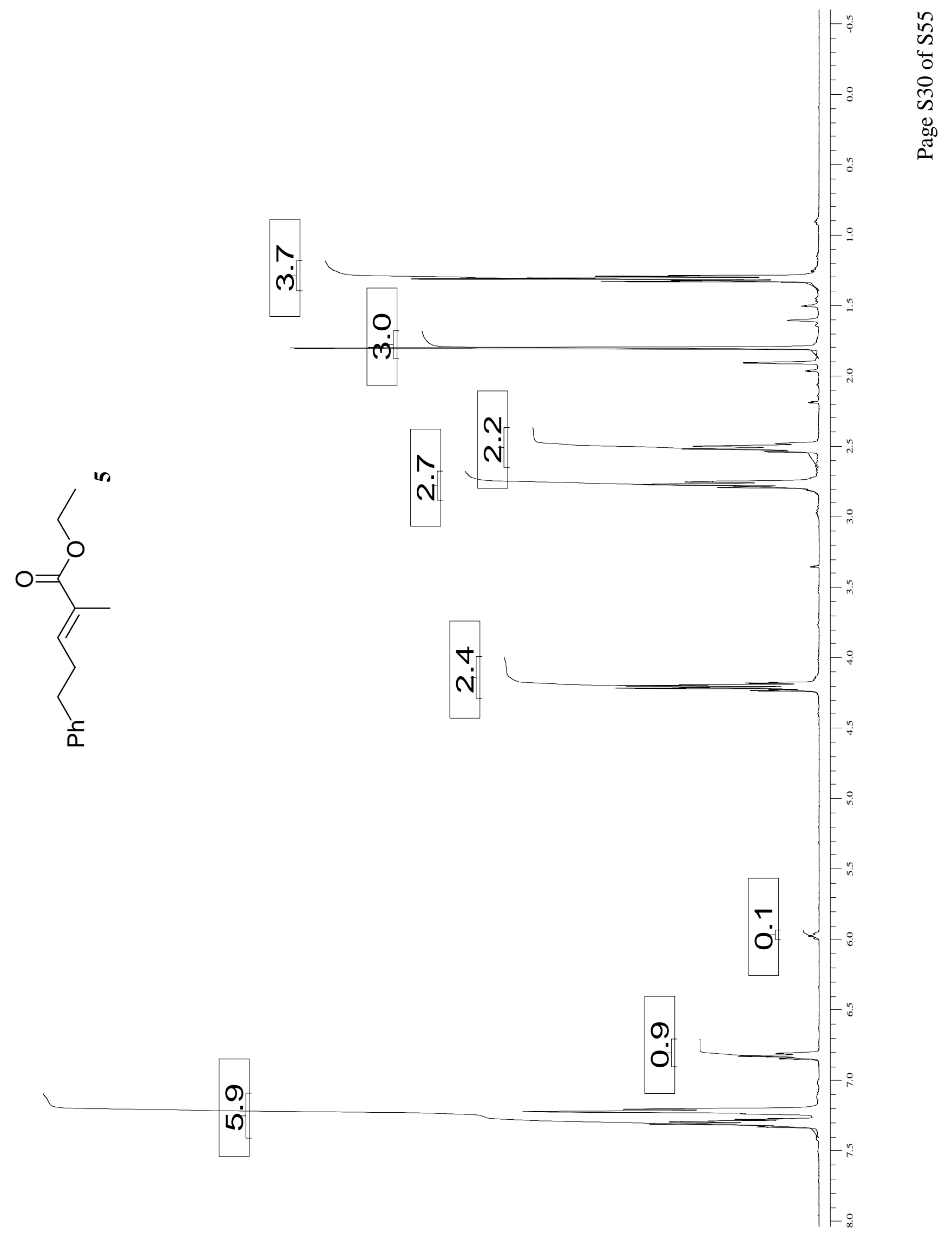




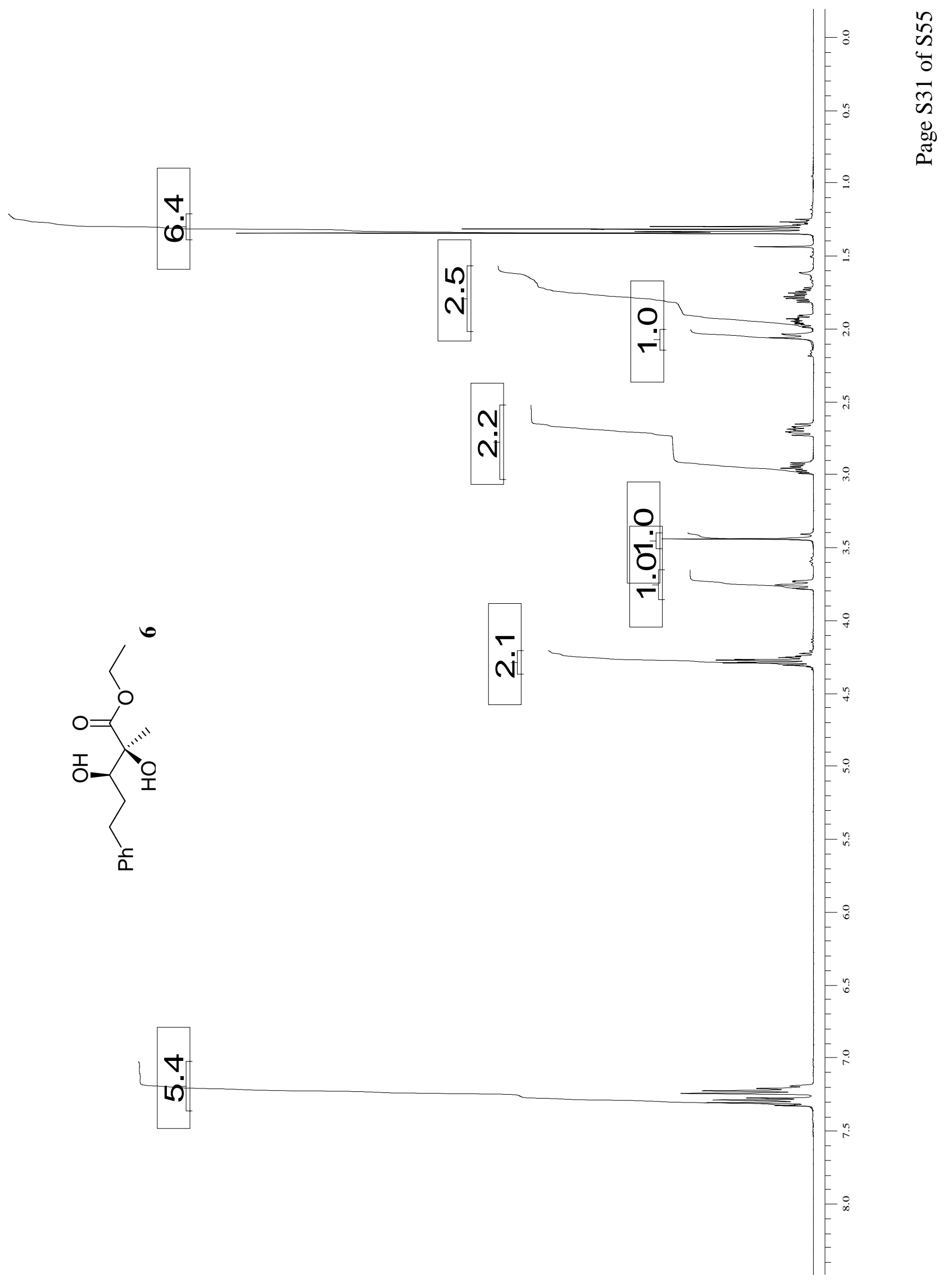




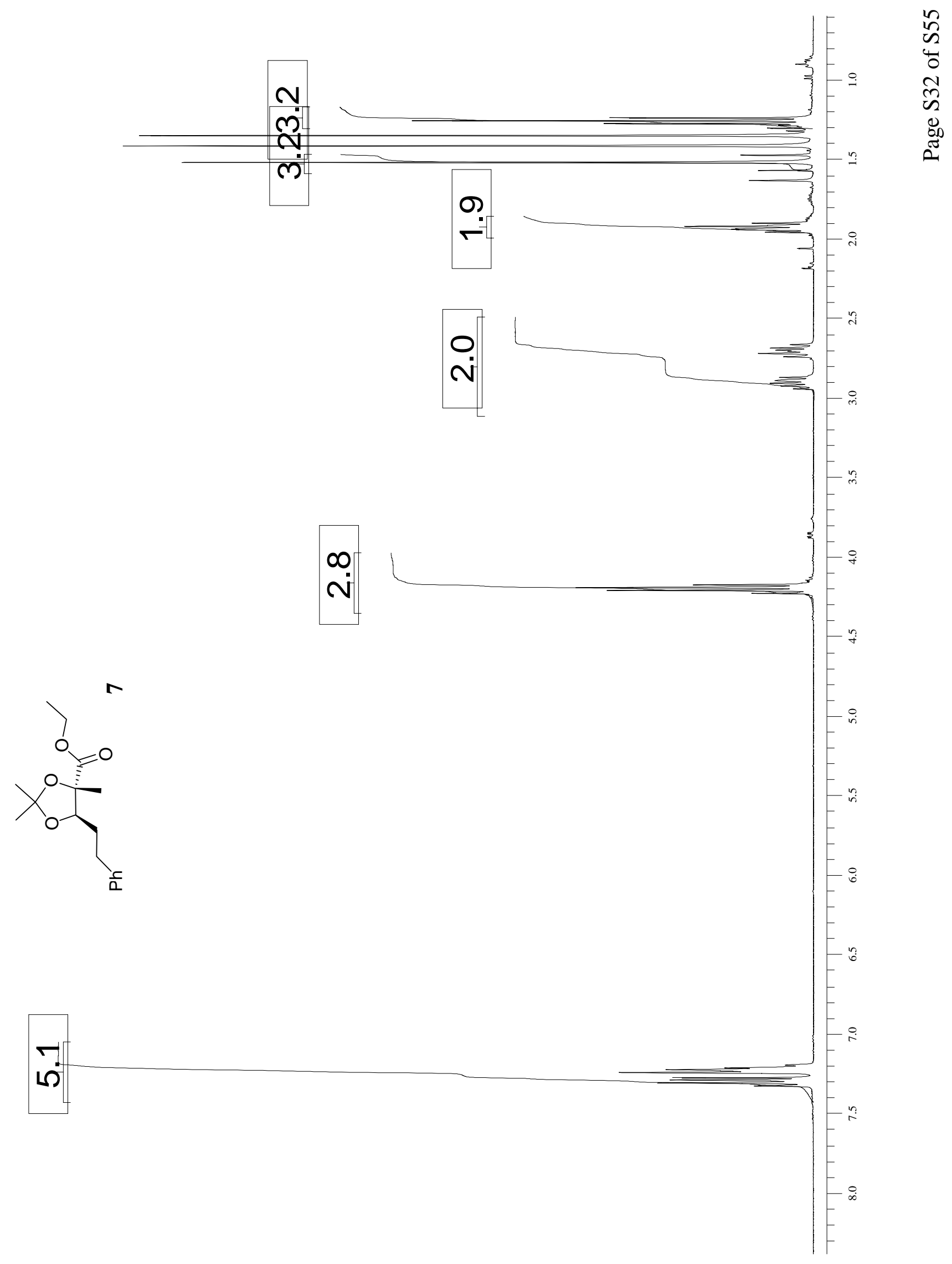




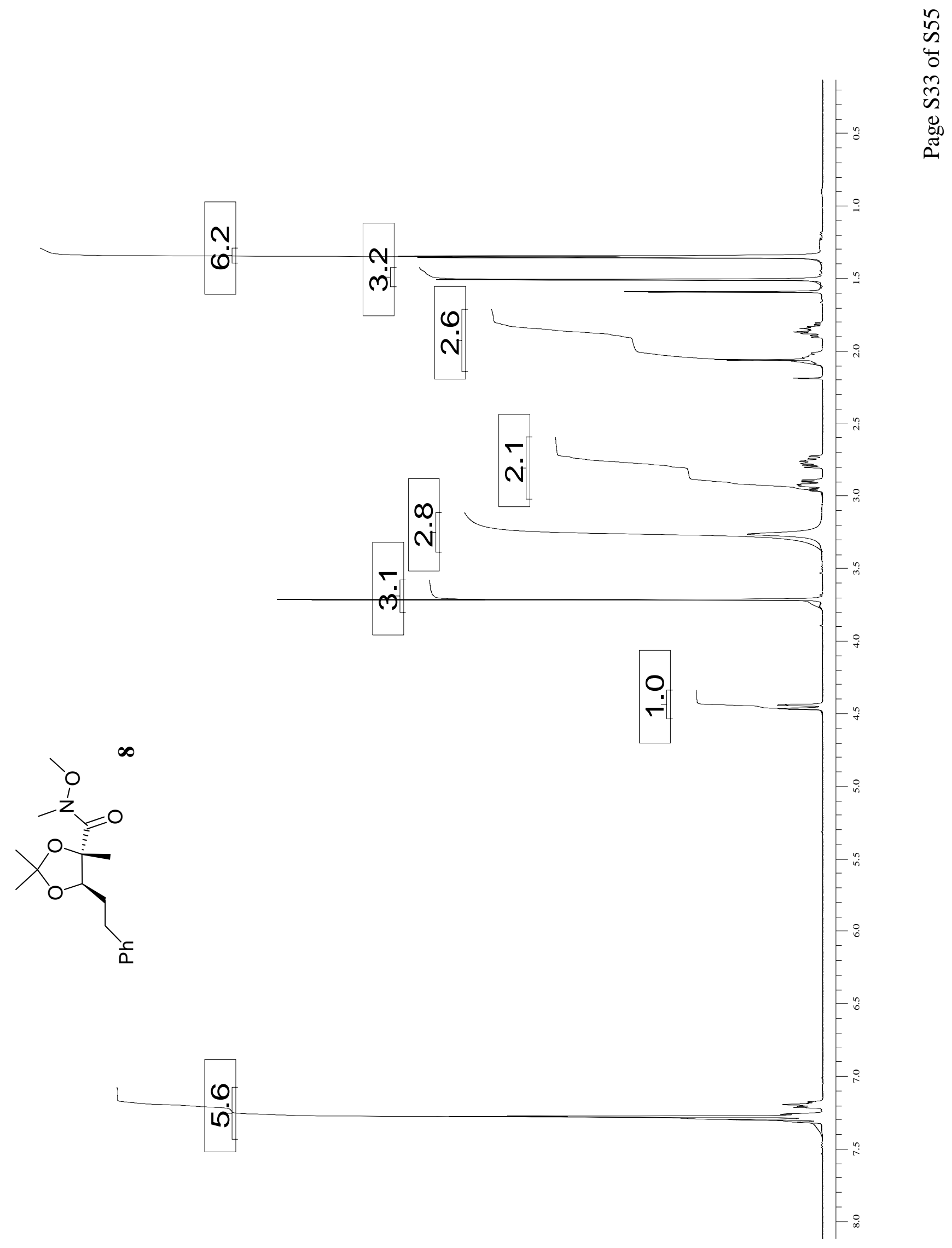




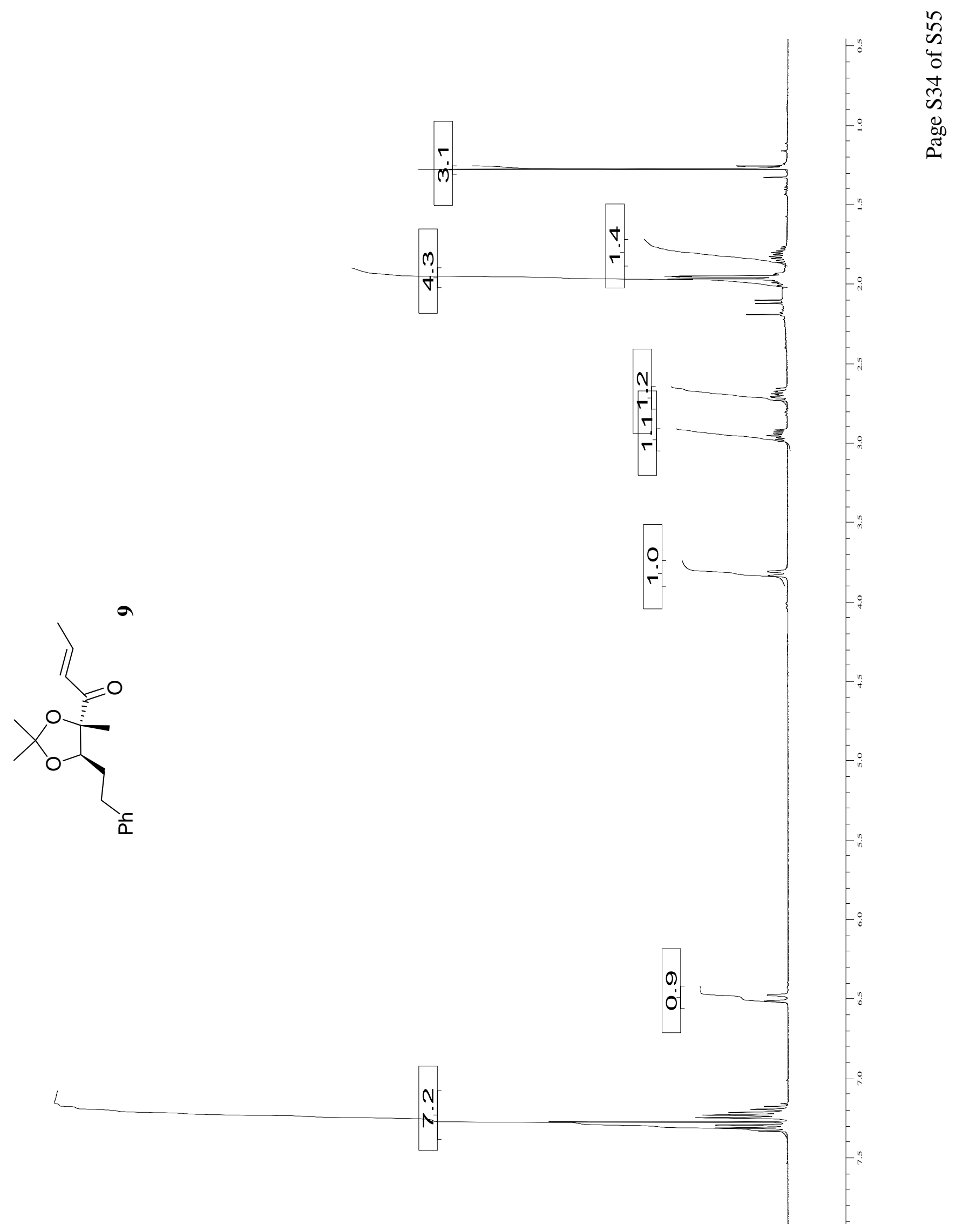




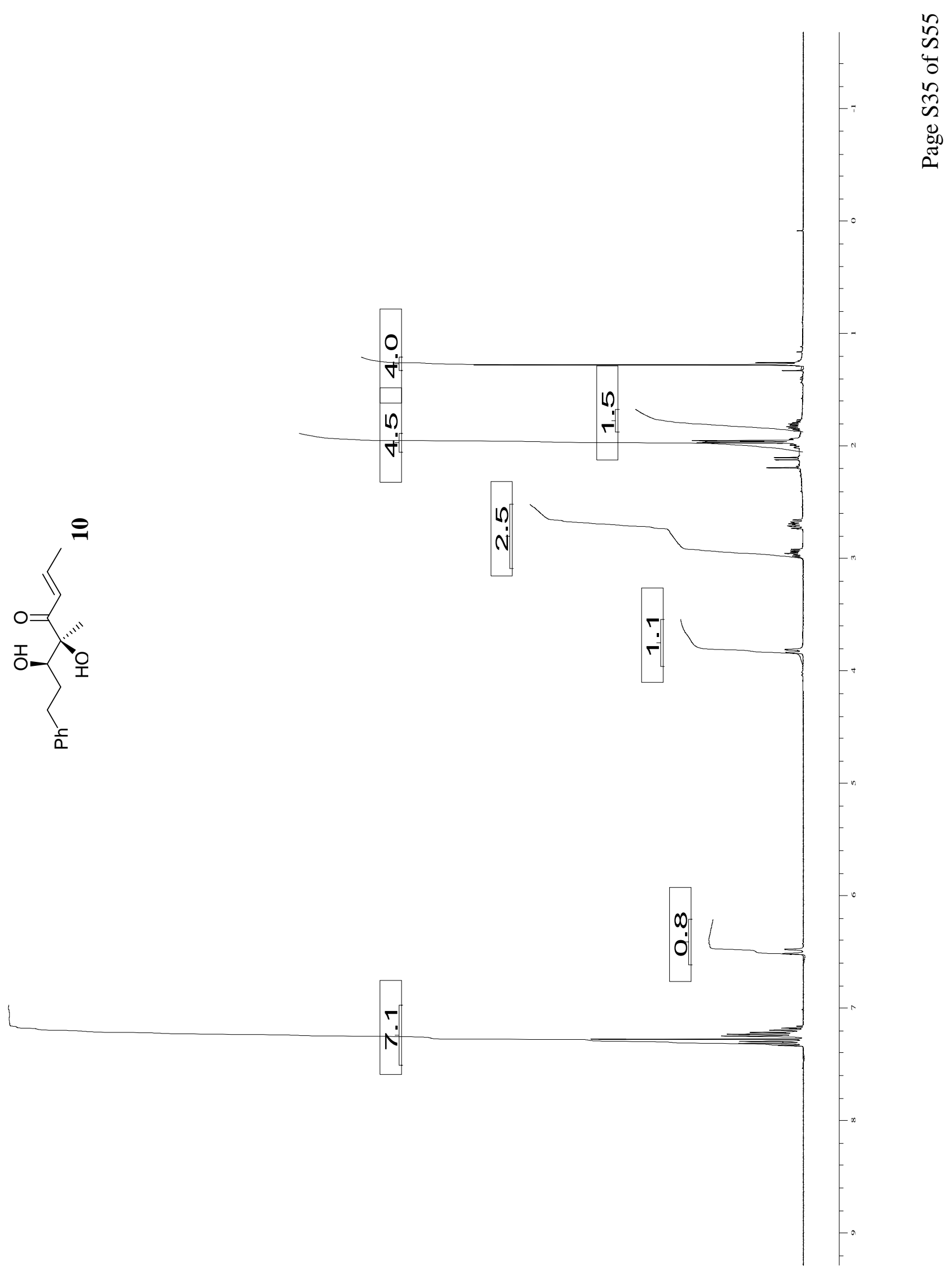




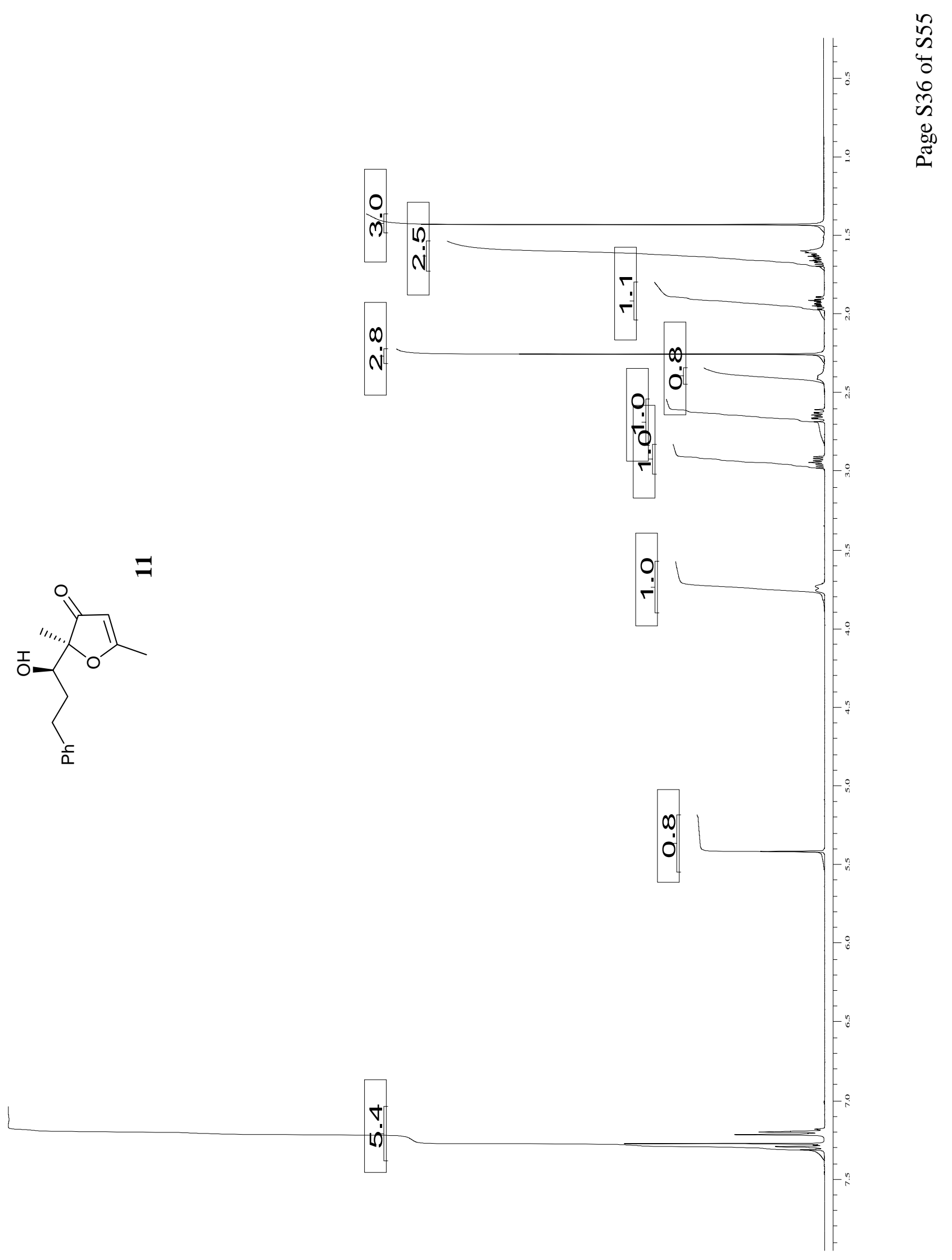




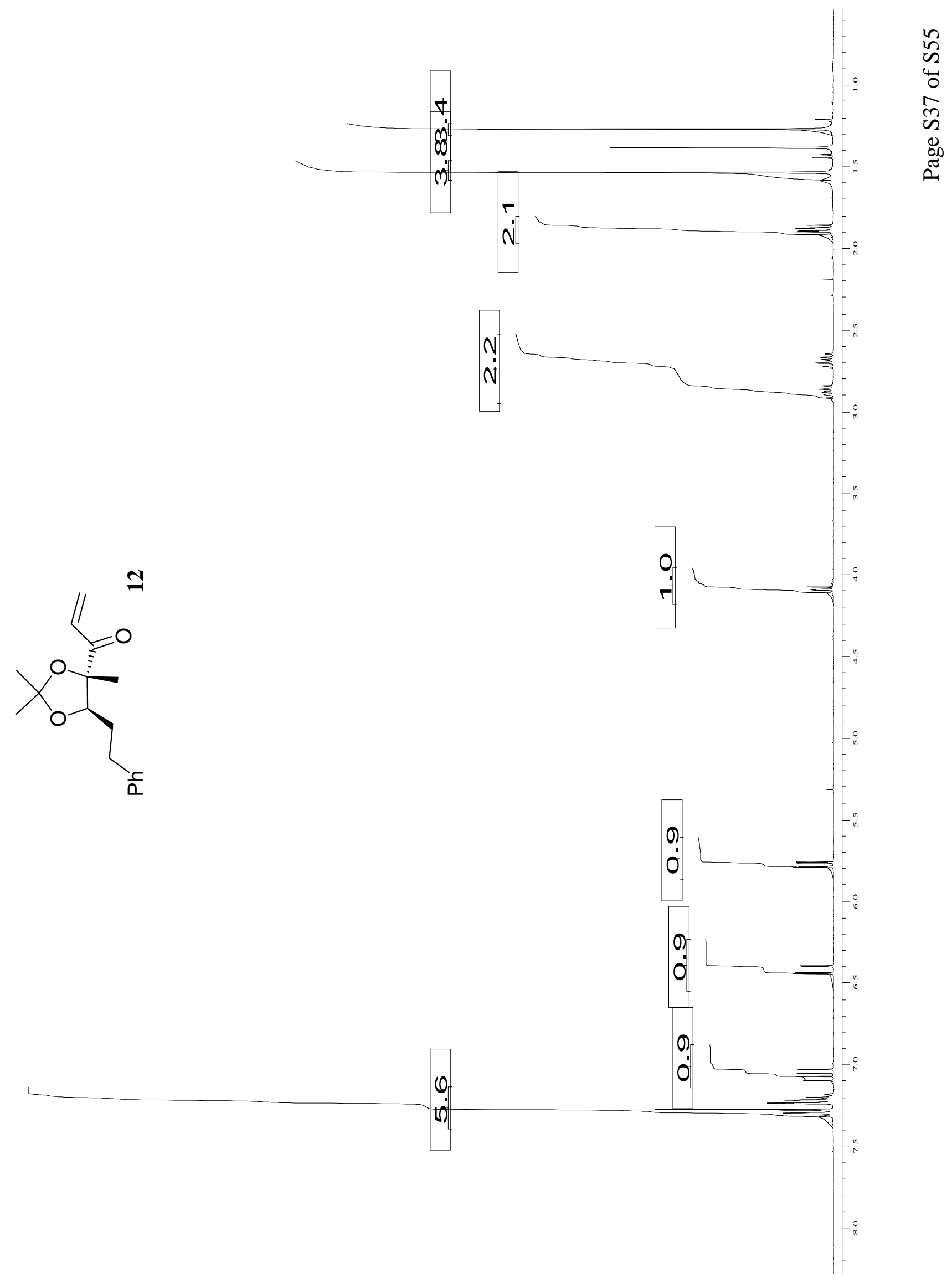




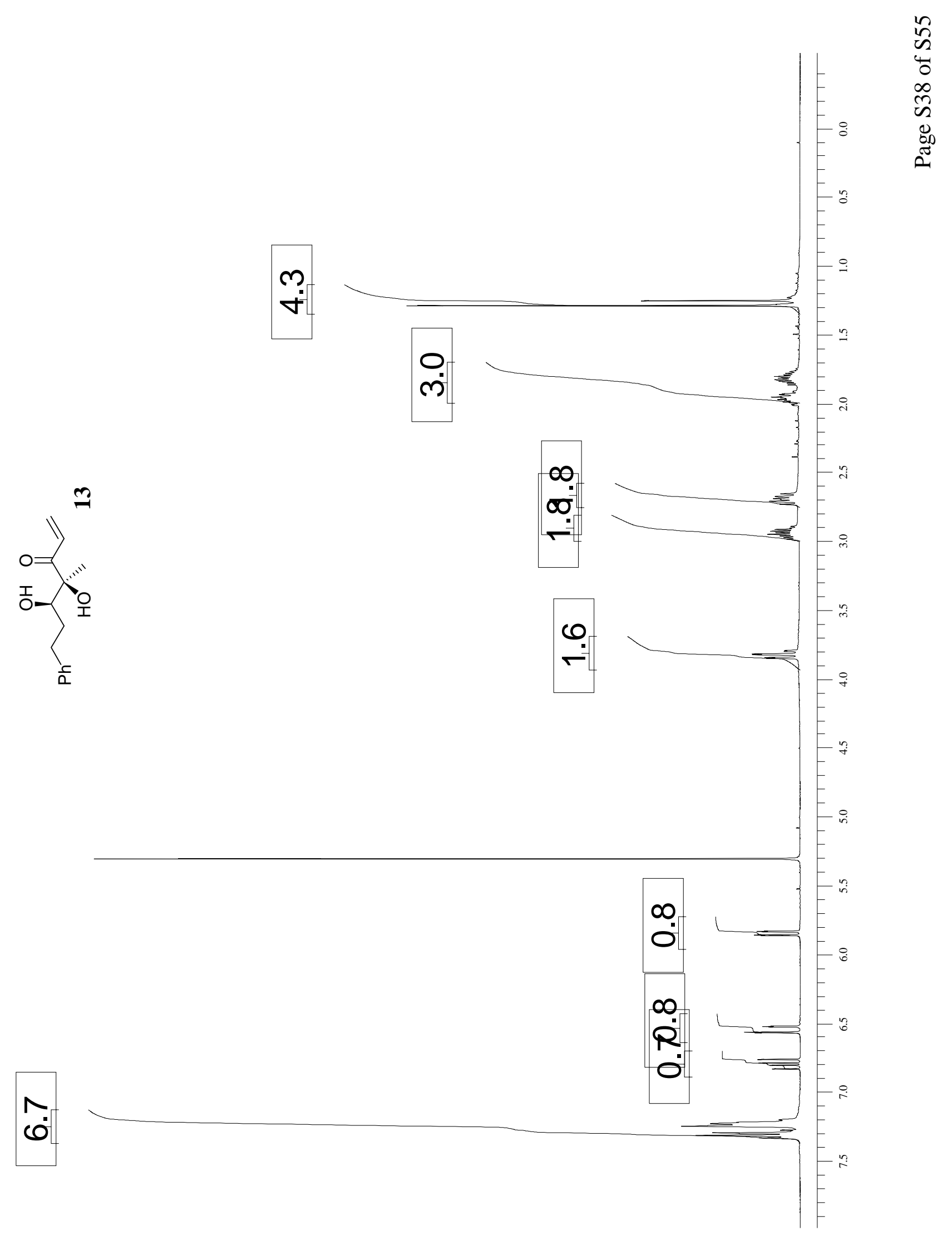




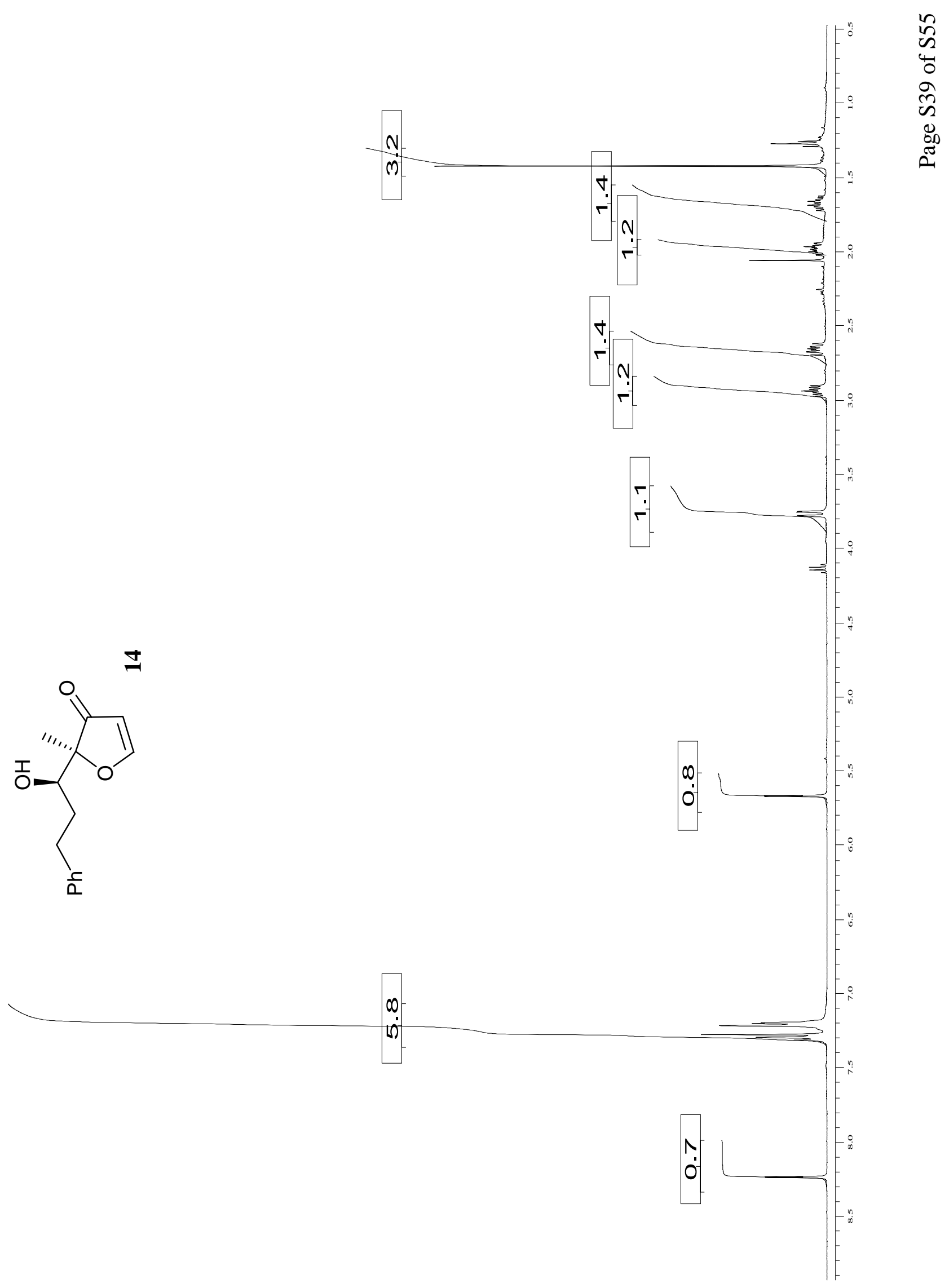




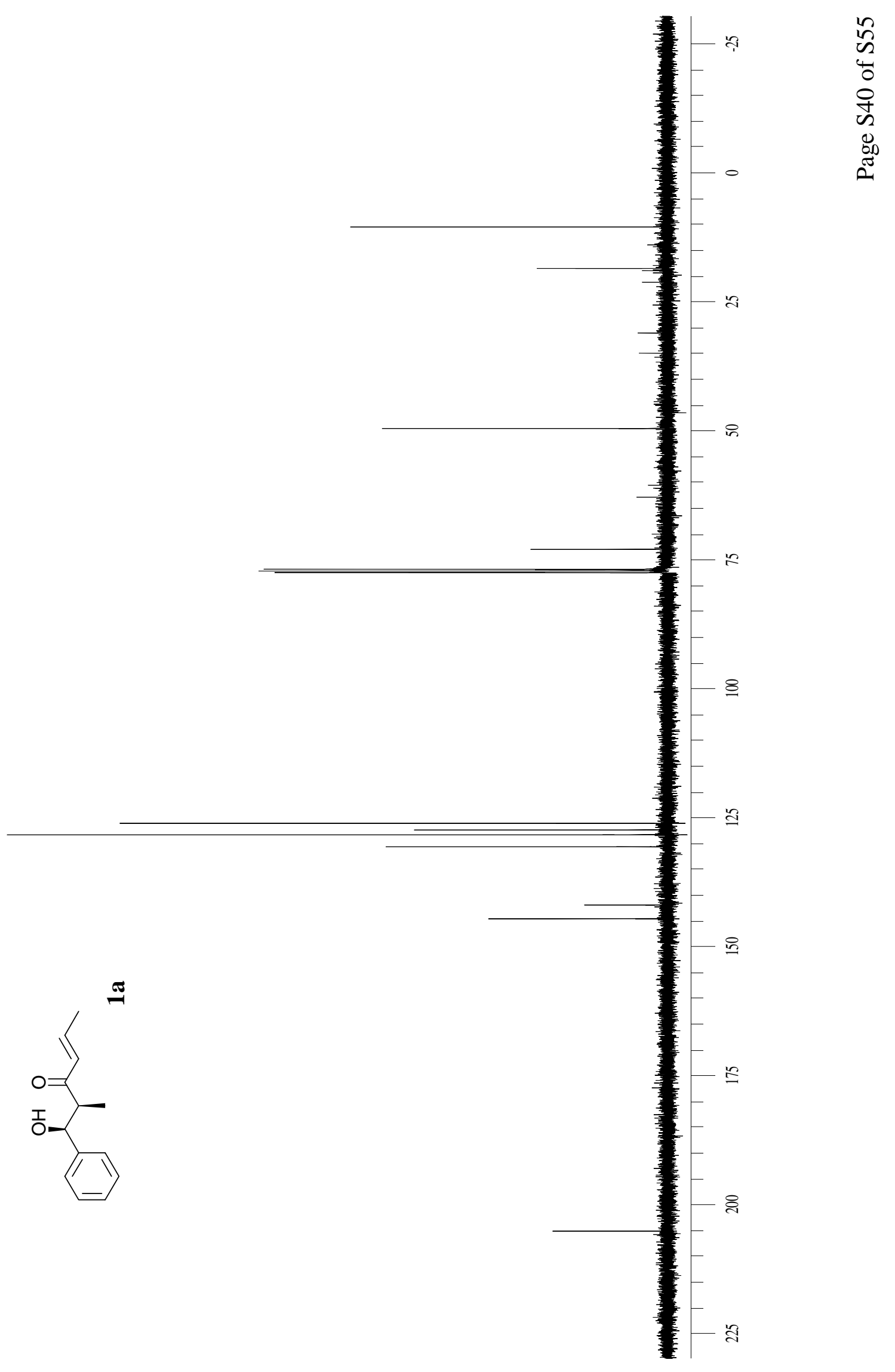




$$
\mid
$$




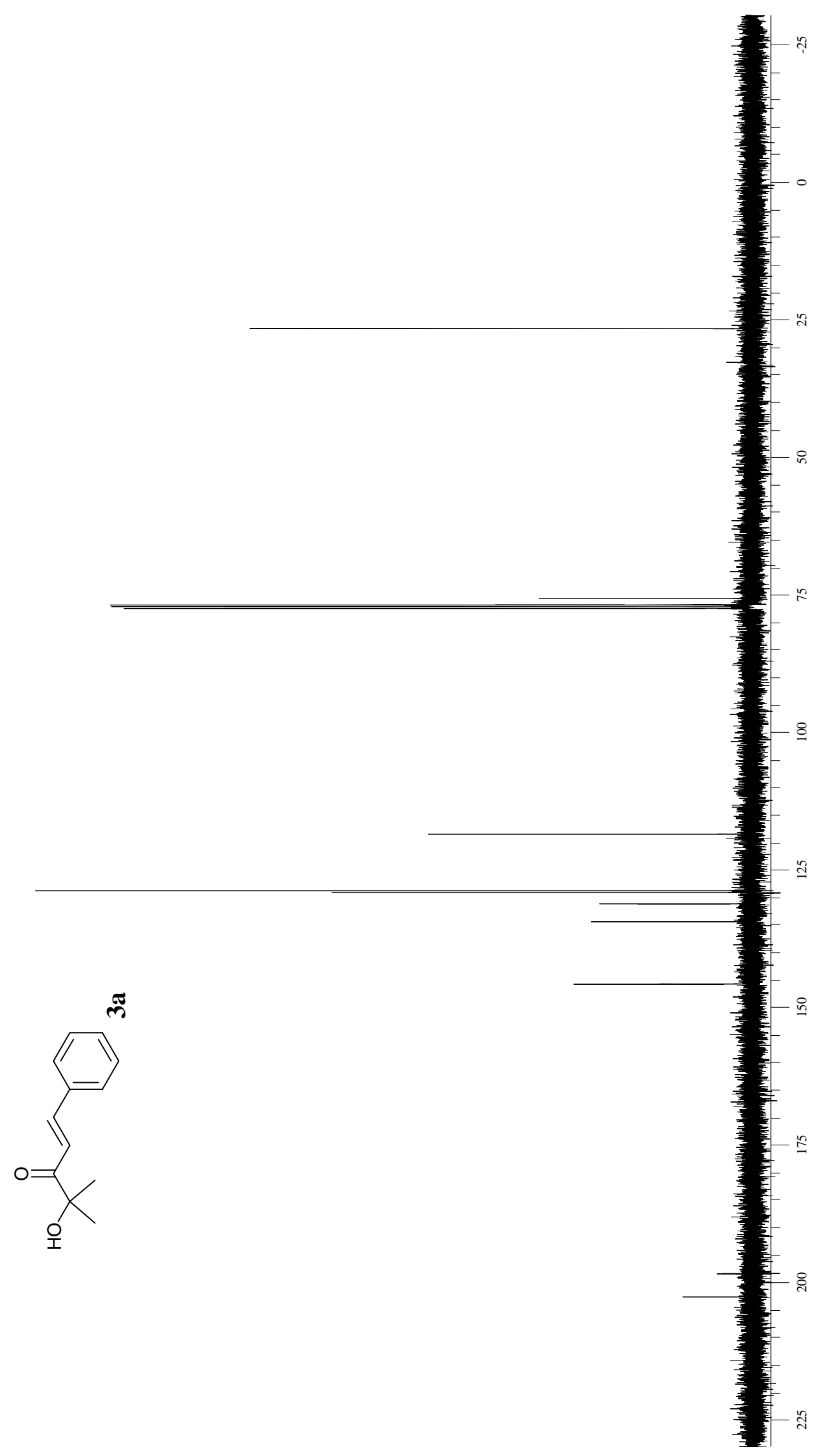

$n$
$n$
$n$
0
0
$y$
$y$
0
0
0
0 


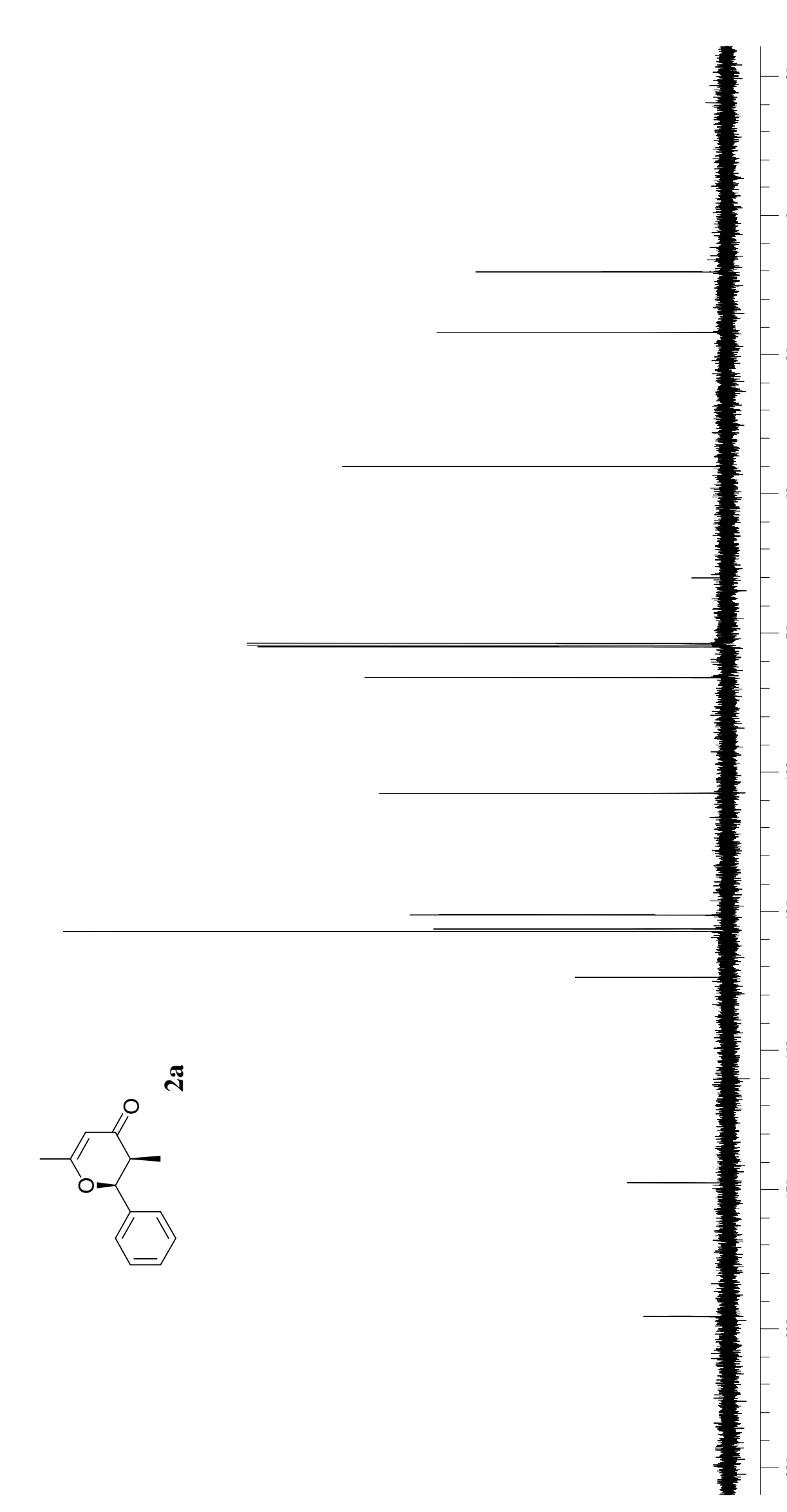




$$
\mid
$$




$$
1
$$




$$
\mid
$$




$$
1
$$




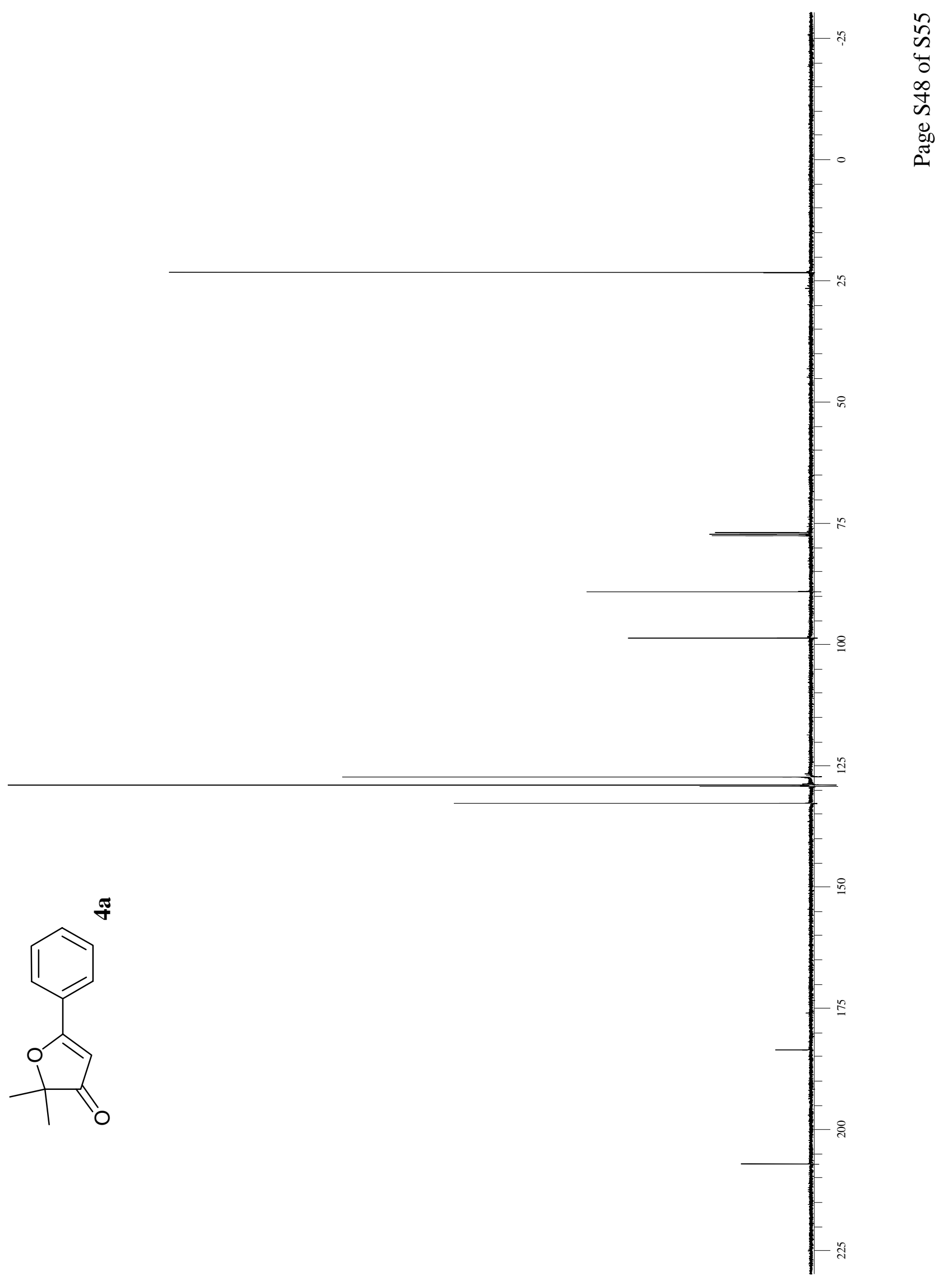




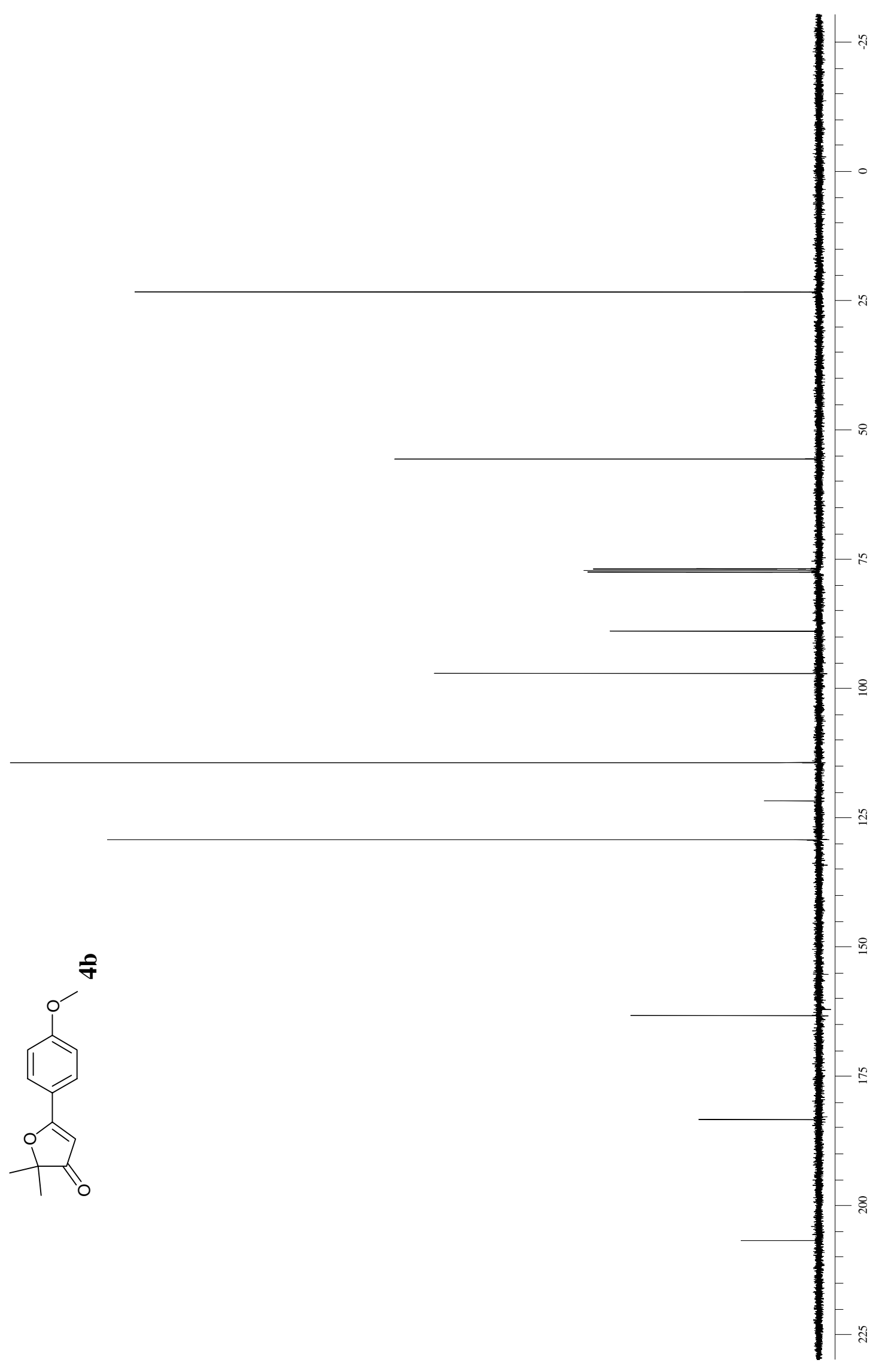

$n$
$n$
$n$
4
0
0
$y$
$w$
0
0
0
0 


$$
1
$$




$$
\mid
$$




$$
\mid
$$




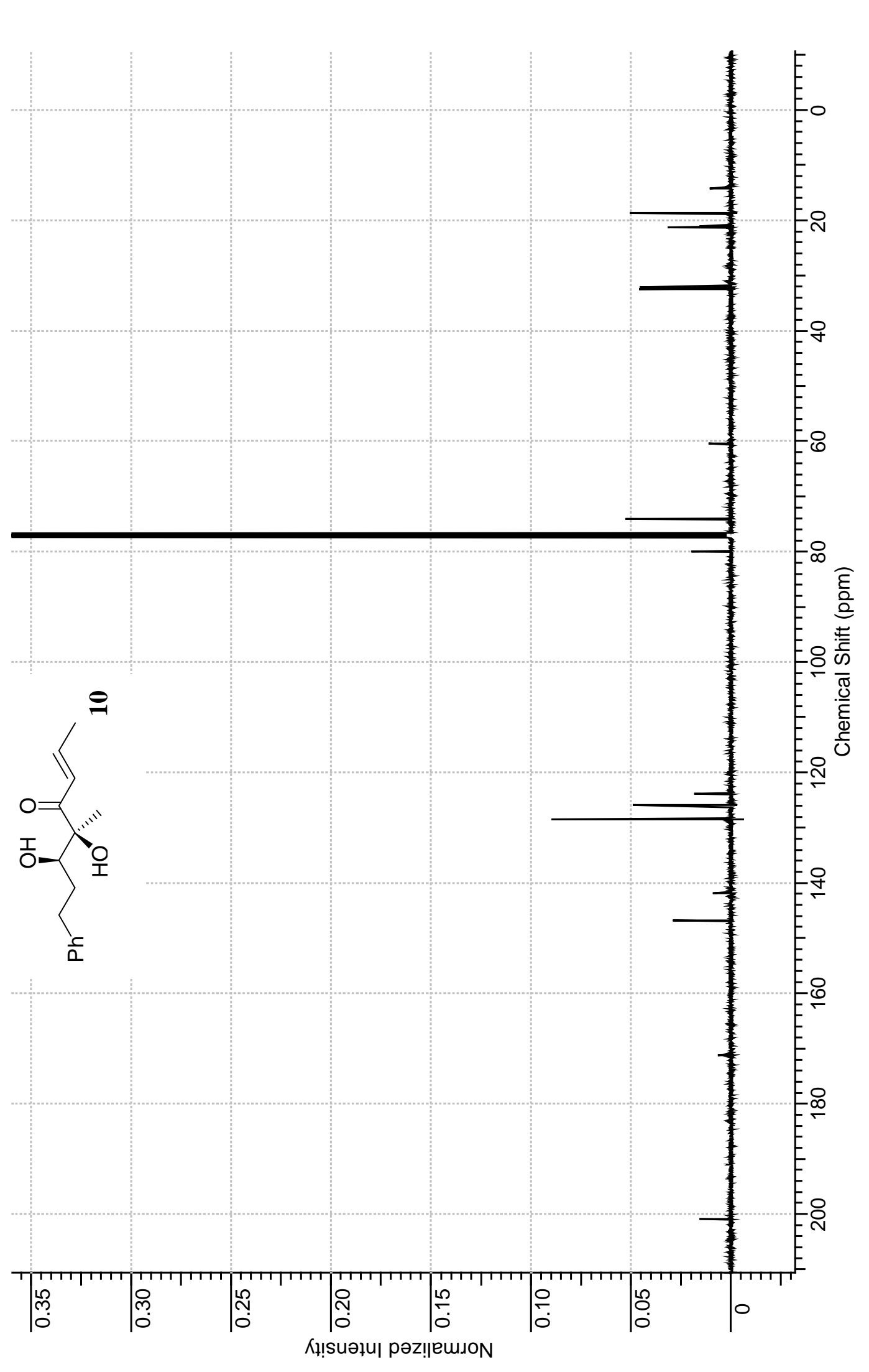

$n$
$n$
$n$
4
$n$
$n$
$n$
0
0
0
0 


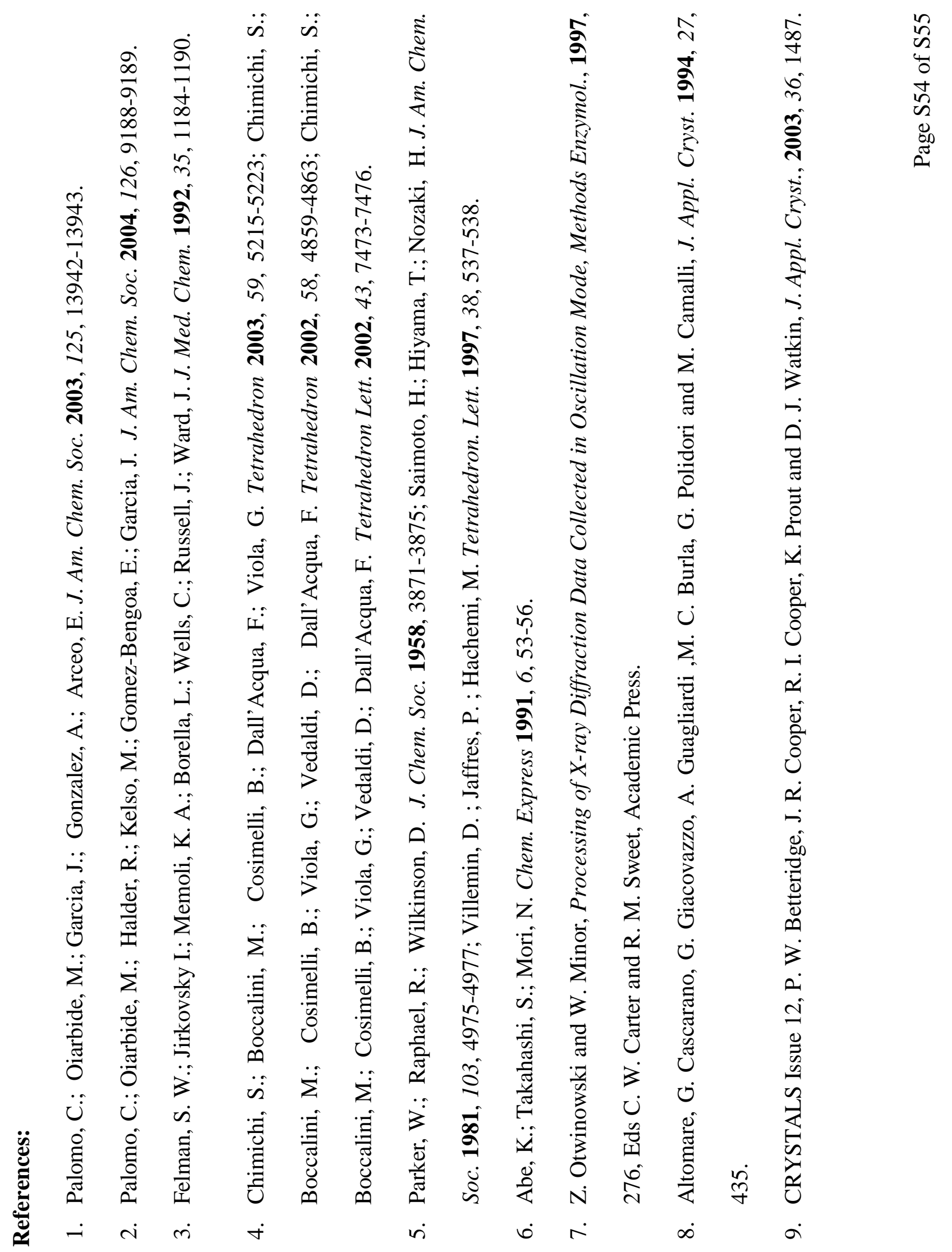


